\title{
Controlled Invariance for Hamiltonian Systems
}

\author{
A. J. van der Schaft \\ Department of Applied Mathematics, Twente University of Technology, The Netherlands
}

\begin{abstract}
A notion of controlled invariance is developed which is suited to Hamiltonian control systems. This is done by replacing the controlled invariant distribution, as used for general nonlinear control systems, by the controlled invariant function group. It is shown how Lagrangian or coisotropic controlled invariant function groups can be made invariant by static, respectively dynamic, Hamiltonian feedback. This constitutes a first step in the development of a geometric control theory for Hamiltonian systems that explicitly uses the given structure.
\end{abstract}

\section{Introduction}

In the last fifteen years the so-called geometric theory of linear systems has proved to be a powerful tool in the solution of various control and synthesis problems (see the trendsetting book of Wonham (1979)). The basic concept in this theory is the notion of controlled invariance or $(A, B)$-invariance of a linear subspace of the state space. Recently, due to the work of Isidori-Krener-Gori-Giorgi-Monaco (1981a) and Hirschorn (1981) this basic notion has been successfully generalized to nonlinear systems (Firstly to nonlinear systems which are affine in the inputs and in Nijmeijer-van der Schaft (1982b) also to general nonlinear systems). In this nonlinear generalization the linear subspaces are replaced by (involutive) distributions on the state space, or their corresponding foliations. Roughly speaking, a distribution on the state space of a system is controlled invariant if it can be made invariant (in a precise geometric sense) by applying (nonlinear) feedback to the system. This notion of a controlled invariant distribution has already been used in problems like nonlinear disturbance decoupling, non-interacting control and invertibility. 
Although the development of this theory has been very successful, it is clear that for many control and synthesis purposes it will not be possible to develop an adequate theory covering all nonlinear systems. For instance the treatment of stability, which is missing up till now in the nonlinear geometric theory, seems very hard in the general case. Therefore it will also be necessary to focus on special types of nonlinear systems. In our opinion, a natural candidate for such a subclass of nonlinear systems is formed by the Hamiltonian systems, as originally proposed by Brockett (1977), and developed in a series of papers by the author and others, see e.g. van der Schaft $(1981,1982,1983 \mathrm{~b}, \mathrm{c})$. A prototype of a Hamiltonian system are the classical Euler-Lagrange equations with external forces. Although in many applications the Hamiltonian description constitutes an idealization (neglection of friction, dissipation etc.) it has proved to be at least a very natural starting point. Outstanding examples are robot manipulators, large space structures and in general (conservative) mechanical systems.

The basic philosophy of this paper is that in dealing with these Hamiltonian systems it is worthwhile to explicitly use the Hamiltonian structure in the solution of control and synthesis problems, and to look for solutions which "remain within the Hamiltonian framework". Most importantly, the feedback which is applied, can and/or should be of a Hamiltonian (and therefore physically interpretable!) form. Of course one could apply the nonlinear geometric theory immediately to Hamiltonian systems. However the feedback which is needed to make a controlled invariant distribution invariant will in general affect the Hamiltonian form of the equations. Since we want to take advantage of the Hamiltonian structure and not to reduce the system to an "ordinary" nonlinear system, this is clearly not satisfying. Therefore as a basic step in building a geometric theory for Hamiltonian systems we have to develop a notion of controlled invariance which is particularly suited to Hamiltonian systems. Preliminary work on such a concept of Hamiltonian controlled invariance has already been done in van der Schaft (1983a) for the case of linear Hamiltonian systems. In this paper this will be extended to the nonlinear case. The basic contribution will be the introduction of the controlled invariant function group, which will replace the controlled invariant distribution. We will prove, under certain conditions, that a Lagrangian controlled invariant function group can be made invariant by Hamiltonian feedback, i.e. feedback which leaves the Hamiltonian form invariant, while coisotropic controlled invariant function groups can be made invariant by dynamic Hamiltonian feedback, i.e. the addition of a Hamiltonian compensator. From a mathematical point of view the notion of a Lagrangian controlled invariant function group is related to the classical concept of complete integrability of Hamiltonian vectorfields.

Certainly, the theory in this paper should be seen as only a first step in the development of a geometric control theory of Hamiltonian systems, and so we will only use Lagrangian and coisotropic controlled invariant function groups in the solution of the somewhat ubiquitous disturbance decoupling problem for Hamiltonian systems. Apart from Lagrangian or coisotropic function groups also symplectic controlled invariant function groups are of much interest. They seem to be the natural tool in the Hamiltonian non-interacting control problem. This will be dealt with in a future paper (Nijmeijer \& van der Schaft (1984c), see also (1984b)). 
Hamiltonian systems. We will briefly review the definition of a Hamiltonian system, see e.g. van der Schaft $(1982,1983 b, c)$. Let $M$ be a $2 n$-dimensional connected manifold with symplectic form $\omega$. By Darboux's theorem there exist local coordinates $(q, p)=\left(q_{1}, \ldots, q_{n}, p_{1}, \ldots, p_{n}\right)$ such that $\omega=\sum_{i=1}^{n} d p_{i} \wedge d q_{i}$. Such coordinates are called canonical. Given a function $F: M \rightarrow \mathbb{R}$ we define the Hamiltonian vectorfield $X_{F}$ on $M$ by $\omega\left(X_{F},-\right)=-d F$. In canonical coordinates

$$
X_{F}=\sum_{i=1}^{n}\left(\frac{\partial F}{\partial p_{i}} \frac{\partial}{\partial q_{i}}-\frac{\partial F}{\partial q_{i}} \frac{\partial}{\partial p_{i}}\right)
$$

Given another function $G: M \rightarrow \mathbb{R}$ we define the Poisson bracket $\{F, G\}=X_{F}(G)$ $=\omega\left(X_{F}, X_{G}\right)$. In canonical coordinates

$$
\{F, G\}=\sum_{i=1}^{n}\left(\frac{\partial F}{\partial p_{i}} \frac{\partial G}{\partial q_{i}}-\frac{\partial F}{\partial q_{i}} \frac{\partial G}{\partial p_{i}}\right)
$$

A (coordinate) transformation $\varphi: M \rightarrow M$ is canonical if $\varphi$ preserves the Poisson bracket, i.e.

$$
\{F \circ \varphi, G \circ \varphi\}=\{F, G\} \circ \varphi \quad \forall F, G
$$

An (affine) Hamiltonian system on $M$ with internal energy $H$ is now defined as

$$
\dot{x}=X_{H}(x)-\sum_{j=1}^{m} u_{j} X_{C_{j}}(x) \quad y_{j}=C_{j}(x)
$$

with $x=(q, p)$ canonical coordinates, $u=\left(u_{1}, \ldots, u_{m}\right)$ the inputs, $y=\left(y_{1}, \ldots, y_{m}\right)$ the outputs, and $C=\left(C_{1}, \ldots, C_{m}\right)$ the output mapping. This constitutes a direct generalization of the classical Euler-Lagrange equations with external forces $u_{i}$

$$
\begin{aligned}
& \frac{d}{d t}\left(\frac{\partial L}{\partial \dot{q}_{i}}\right)-\frac{\partial L}{\partial q_{i}}= \begin{cases}u_{i} & i=1, \ldots, m \\
0 & i=m+1, \ldots, n\end{cases} \\
& y_{j}=q_{j} \\
& j=1, \ldots, m
\end{aligned}
$$

or in Hamiltonian form (with $p_{i}=\frac{\partial L}{\partial \dot{q}_{i}}$ the momenta and $H(q, p)=\sum_{i=1}^{n} \dot{q}_{i} \frac{\partial L}{\partial \dot{q}_{i}}-$ $L(q, \dot{q})$ the internal energy)

$$
\begin{array}{ll}
\dot{q}_{i}=\frac{\partial H}{\partial p_{i}} & i=1, \ldots, n \\
\dot{p}_{i}=-\frac{\partial H}{\partial q_{i}}+u_{i} & i=1, \ldots, m \\
\dot{p}_{i}=-\frac{\partial H}{\partial q_{i}} & i=m+1, \ldots, n \\
y_{j}=q_{j} & j=1, \ldots, m
\end{array}
$$


In fact, if we take $C_{j}(q, p)=q_{j}$ in (1.4) we obtain (1.6), and conversely if we allow for canonical coordinate transformations on $(q, p)$ in $(1.6)$, then (1.6) becomes of the form (1.4).

If we interpret $y=\left(y_{1}, \ldots, y_{m}\right)$ as coordinates for an $m$-dimensional output manifold $Y$, then $\left(y_{1}, \ldots, y_{m}, u_{1}, \ldots, u_{m}\right)$ can be most naturally interpreted as natural coordinates for the cotangent bundle $T^{*} Y$. (If $\left(y_{1}, \ldots, y_{m}\right)$ are arbitrary coordinates for $Y$, then we define natural coordinates $\left(y_{1}, \ldots, y_{m}, u_{1}, \ldots, u_{m}\right)$ for $T^{*} Y$ by letting a point $\left(\bar{y}_{1}, \ldots, \bar{y}_{m}, \bar{u}_{1}, \ldots, \bar{u}_{m}\right)$ in these coordinates correspond to the one-form $\sum_{j=1}^{m} \bar{u}_{j} d y_{j}$ on $Y$ in the point $\left(\bar{y}_{1}, \ldots, \bar{y}_{m}\right)$.) Being a cotangent bundle $T^{*} Y$ has a natural symplectic form $\omega_{m}^{e}$. In fact, if $\left(y_{1}, \ldots, y_{m}, u_{1}, \ldots, u_{m}\right)$ are natural coordinates, then $\omega^{e}$ equals $\sum_{j=1}^{m} d u_{j} \wedge d y_{j}$. So natural coordinates are always canonical. Notice that if we choose another set of coordinates $\left(y_{1}^{\prime}, \ldots, y_{m}^{\prime}\right)$ for $Y$, then the coordinates $\left(u_{1}, \ldots, u_{m}\right)$ have to change to $\left(u_{1}^{\prime}, \ldots, u_{m}^{\prime}\right)$ in such a way that $\omega^{e}=\sum_{j=1}^{m} d u_{j}^{\prime} \wedge d y_{j}^{\prime}$. For instance, if we transform the outputs from Cartesian to angular coordinates, then the inputs change from translational forces to torques.

Function groups. We briefly collect some facts about function groups and Poisson structures, which date back to Lie (1890) and were recently rediscovered by various authors (cf. Weinstein (1983), Hermann (1977)). Let $M$ be a connected symplectic manifold with Poisson bracket $\{F, G\}=\omega\left(X_{F}, X_{G}\right)$. We call a collection $\mathscr{F}$ of smooth functions from $M$ to $\mathbb{R}$ a function space, if

1) $\mathscr{F}$ is a linear subspace (over $\mathbb{R}$ ) of $C^{\infty}(M)$, the smooth functions on $M$.

2) If $F_{1}, \ldots, F_{s} \in \mathscr{F}$ and $G: \mathbb{R}^{s} \rightarrow \mathbb{R}$ is a smooth function, then $G\left(F_{1}, \ldots, F_{s}\right)$ $\in \mathscr{F}$. Furthermore, we call $\mathscr{F}$ a function group if also

3) $\mathscr{F}$ is closed under Poisson bracket, i.e. if $F_{1}, F_{2} \in \mathscr{F}$, then $\left\{F_{1}, F_{2}\right\} \in \mathscr{F}$.

Notice that by 2) a non-empty function space always contains $\mathbb{R}$, the constant functions on $M$ (actually by this fact condition 2) implies condition 1)!). Given some functions $F_{1}, \ldots, F_{k}$ on $M$ we denote by span $\left\{F_{1}, \ldots, F_{k}\right\}$ the smallest function space containing these functions. Furthermore the sum $\mathscr{F}^{1}+\mathscr{F}^{2}$ of two function spaces $\mathscr{F}^{-1}, \mathscr{F}^{2}$ will be the smallest function space containing $\mathscr{F}^{1}$ as well as $\mathscr{F}^{2}$. Given a function space $\mathscr{F}$, we denote by $\overline{\mathscr{F}}$ the closure of $\mathscr{F}$ under Poisson bracket, i.e. the smallest function group containing $\mathscr{F}$. Furthermore we define

$$
\mathscr{F}^{\perp}=\left\{G \in C^{\infty}(M) \mid\{G, F\}=0, \forall F \in \mathscr{F}\right\}
$$

Let now $G_{1}, G_{2} \in \mathscr{F}^{\perp}$ and $K: \mathbb{R}^{2} \rightarrow \mathbb{R}$, then

$$
\left\{K\left(G_{1}, G_{2}\right), F\right\}=\frac{\partial K}{\partial x_{1}}\left(G_{1}, G_{2}\right)\left\{G_{1}, F\right\}+\frac{\partial K}{\partial x_{2}}\left(G_{1}, G_{2}\right)\left\{G_{2}, F\right\}=0
$$


and hence $\mathscr{F}^{\perp}$ is a function space. Furthermore by the Jacobi-identity

$$
\left\{\left\{G_{1}, G_{2}\right\}, F\right\}+\left\{\left\{G_{2}, F\right\}, G_{1}\right\}+\left\{\left\{F, G_{1}\right\}, G_{2}\right\}=0
$$

for any $G_{1}, G_{2} \in \mathscr{F}^{\perp}$ and $F \in \mathscr{F}$. Hence $\left\{G_{1}, G_{2}\right\} \in \mathscr{F}^{\perp}$ and $\mathscr{F}^{\perp}$ is actually a function group, called the polar group. Finally $\mathscr{F} \cap \mathscr{F}^{\perp}$ is a function space (resp. group) if $\mathscr{F}$ is a function space (resp. group), and the elements of $\mathscr{F} \cap \mathscr{F} \perp$ are called the distinguished or Casimir functions. It is clear that for any function space $\mathscr{F}$

$$
\mathscr{F} \cap \mathscr{F}^{\perp} \subset \mathscr{F} \subset \bar{F} \subset\left(\mathscr{F}^{\perp}\right)^{\perp}
$$

and for any two function spaces $F_{1}, F_{2}$

$$
\begin{aligned}
& \mathscr{F}_{1}+\mathscr{F}_{2} \subset \overline{\mathscr{F}_{1}+\mathscr{F}_{2}} \subset\left(\mathscr{F}_{1}^{\perp} \cap \mathscr{F}_{2}{ }^{\perp}\right)^{\perp} \\
& \mathscr{F}_{1} \cap \mathscr{F}_{2} \subset \overline{\mathscr{F}_{1} \cap \mathscr{F}_{2}} \subset\left(\mathscr{F}_{1}{ }^{\perp}+\mathscr{F}_{2}{ }^{\perp}\right)^{\perp}
\end{aligned}
$$

For a function space $\mathscr{F}$ we define the codistribution $d \mathscr{F}$ as

$$
d \mathscr{F}(x)=\operatorname{span}_{\mathbf{R}}\{d \mathscr{F}(x) \mid F \in \mathscr{F}\}, x \in M
$$

and the distribution $D_{\mathscr{F}}$ as

$$
D_{\mathscr{F}}(x)=\operatorname{span}_{\mathbf{R}}\left\{X_{F}(x) \mid F \in \mathscr{F}\right\}, x \in M
$$

In order to simplify considerably the technical details of the sequel we make the following assumption (also dating back to Lie), which will hold throughout this paper.

Assumption 1. Every function space satisfies

Condition A. There exists a number of independent functions $F_{1}, \ldots, F_{k}$ on $M$ such that $\mathscr{F}=\operatorname{span}\left\{F_{1}, \ldots, F_{k}\right\}$ (independent means that $\operatorname{dim}$ $\left.\operatorname{span}_{\mathbf{R}}\left\{d F_{1}(x), \ldots, d F_{k}(x)\right\}=k, \forall x\right)$.

Renzark. In applications this assumption may not be the most natural one. Instead, one may replace condition A by the weaker

Condition $\mathbf{A}^{\prime} . \quad \operatorname{dim} d \mathscr{F}(x)=$ constant, $\forall x$

If Condition $\mathrm{A}^{\prime}$ is satisfied, there exist locally $k$ independent functions such that locally $\mathscr{F}=\operatorname{span}\left\{F_{1}, \ldots, F_{k}\right\}$. However this implies that some of the following propositions (especially Lemma 1.2 and its consequences) hold only locally.

With every function group satisfying Condition $\mathrm{A}$ we can associate a so-called Poisson structure. Consider a set of smooth functions $w_{i j}, i, \dot{j}=1, \ldots, k$ on $\mathbb{R}^{k}$. 
They define a Poisson structure if (cf. Weinstein (1983))
i) $\quad w_{i j}+w_{j i}=0$
$i, j=1, \ldots, k$
ii)

$$
\sum_{l=1}^{k}\left(w_{l j} \frac{\partial w_{i r}}{\partial x_{l}}+w_{l i} \frac{\partial w_{r j}}{\partial x_{l}}+w_{l r} \frac{\partial w_{j i}}{\partial x_{l}}\right)=0
$$$$
i, j, r=1, \ldots, k
$$

By i) the rank of the matrix $\left(w_{i j}(x)\right)$ is for every $x$ even. If rank $\left(w_{i j}(x)\right)=k$, so $k=2 n$, for every $x$, then we speak of a symplectic structure. In fact in this case $\omega:=\sum_{i, j=1}^{2 n} w^{i j}(x) d x_{i} \wedge d x_{j}$, where $\left(w^{i j}(x)\right)=\left(w_{i j}(x)\right)^{-1}$, is a symplectic form on $\mathbb{R}^{2 n}$ (this follows from i) and ii)).

Given a Poisson structure $w_{i j}$ on $\mathbb{R}^{k}$ and a function $F$ on $\mathbb{R}^{k}$ we define the Hamiltonian vectorfield $X_{F}$ on $\mathbb{R}^{k}$ by

$$
X_{F}(x)=-\sum_{i, j=1}^{k} w_{i j}(x) \frac{\partial F(x)}{\partial x_{j}} \frac{\partial}{\partial x_{i}}
$$

It is easy to see that in case $w_{i j}$ is a symplectic structure this is just the ordinary definition of a Hamiltonian vectorfield as in (1.1). Moreover we define a Poisson bracket $\{,\}_{\mathbf{R}^{k}}$ corresponding to the Poisson structure $w_{i j}$ on $\mathbb{R}^{k}$ as follows. Let $F, G: \mathbb{R}^{k} \rightarrow \mathbb{R}$, then

$$
\{F, G\}_{\mathbf{R}^{k}}(x)=\sum_{i, j=1}^{k} w_{i j}(x) \frac{\partial F}{\partial x_{i}}(x) \frac{\partial G}{\partial x_{j}}(x)
$$

Again it is easy to conclude that if $w_{i j}$ is a symplectic structure then this is just the ordinary Poisson bracket (1.2) on $\mathbb{R}^{2 n}$. It follows from i) and ii) that $\{,\}_{\mathbf{R}^{k}}$ is anti-symmetric and satisfies the Jacobi-identity

$$
\left\{\{F, G\}_{\mathbf{R}^{k}}, H\right\}_{\mathbf{R}^{k}}+\left\{\{G, H\}_{\mathbf{R}^{k}}, F\right\}_{\mathbf{R}^{k}}+\left\{\{H, F\}_{\mathbf{R}^{k}}, G\right\}_{\mathbf{R}^{k}}=0
$$

as the ordinary Poisson bracket.

The connection between Poisson structures and function groups satisfying Condition $\mathrm{A}$ is the following. Let $\mathscr{F}=\operatorname{span}\left\{F_{1}, \ldots, F_{k}\right\}$ be a function group on $(M, \omega)$, with $F_{i}$ independent. Then there exist functions $w_{i j}: \mathbb{R}^{k} \rightarrow \mathbb{R}, i, j=$ $1, \ldots, k$, such that

$$
\left\{F_{i}, F_{j}\right\}_{M}=w_{i j} \circ\left(F_{1}, \ldots, F_{k}\right)
$$

It follows from the properties of the usual Poisson bracket on $(M, \omega)$ that the functions $w_{i j}$ satisfy i) and ii). Hence a function group satisfying Condition $\mathrm{A}$ defines a Poisson structure! Using the theory of Poisson structures one can prove the following basic theorem on function groups (Lie (1890), Weinstein (1983)).

Theorem 1.1. Let $\mathscr{F}$ be a function group on $(M, \omega)$ satisfying Condition A such that $d\left(\mathscr{F} \cap \mathscr{F}^{\perp}\right)(x)$ has constant dimension. Suppose $\operatorname{dim} d \mathscr{F}=k$ and $\operatorname{dim} d(\mathscr{F}$ 
$\left.\cap \mathscr{F}^{\perp}\right)=r$. Then locally there exist canonical coordinates $\left(q_{1}, \ldots, q_{n}, p_{1}, \ldots, p_{n}\right)$ for $M$ such that

$$
F=\operatorname{span}\left\{q_{1}, \ldots, q_{l}, p_{1}, \ldots, p_{l}, p_{l+1}, \ldots, p_{l+r}\right\}
$$

with $2 l+r=k$.

Remark. Since the above theorem is local, it remains valid if we replace Condition A by Condition $\mathrm{A}^{\prime}$.

We will now derive some propositions which will be useful later on. First we derive some connections between $\mathscr{F}$ and its distribution $D_{\mathscr{F} \text {. }}$

Lemma 1.2. Let $\mathscr{F}$ be a function space satisfying Condition $\mathrm{A}$ and let $G: M \rightarrow \mathbb{R}$ be such that $d G \in d \mathscr{F}$ (i.e. $d G(x) \in d \mathscr{F}(x), \forall x)$. Then $G \in \mathscr{F}$. Also if $X_{G} \in D_{\mathscr{F}}$, then $G \in \mathscr{F}$.

Proof. By Condition A, $\mathscr{F}=\operatorname{span}\left\{F_{1}, \ldots, F_{k}\right\}$, with $F_{i}$ independent functions. Denote the map $\left(F_{1}, \ldots, F_{k}\right): M \rightarrow \mathbb{R}^{k}$ by $F$. Then since $d G \in d \mathscr{F}$, there exists a 1-form $\alpha_{G}$ on $\mathbb{R}^{k}$ such that $F^{*} \alpha_{G}=d G$. Hence $F^{*}\left(d \alpha_{G}\right)=d(d G)=0$ and so $d \alpha_{G}=0$. By Poincare's lemma there exists a function $G: \mathbb{R}^{k} \rightarrow \mathbb{R}$ such that $\alpha_{G}=d \tilde{G}$. Therefore $d\left(F^{*} \circ \tilde{G}\right)=d G$, or equivalently, $d\left(\tilde{G} \circ\left(F_{1}, \ldots, F_{k}\right)-G\right)=0$. Since $M$ is connected (and hence pathwise connected) this implies that $G=$ $\tilde{G} \circ\left(F_{1}, \ldots, F_{k}\right)+$ constant. Since the constant functions are included in $\mathscr{F}$ we obtain $G \in \mathscr{F}$. Finally, if $X_{G} \in D_{\mathscr{F}}$, then $d G=-\omega\left(X_{G},-\right) \in d \mathscr{F}$, and hence $G \in \mathscr{F}$.

A (general) distribution $D$ on $M$ is called involutive if whenever $X_{1}$ and $X_{2}$ are vectorfields in $D$ (i.e. $X_{i}(x) \in D(x), \forall x, i=1,2$ ), then also the Lie bracket $\left[X_{1}, X_{2}\right]$ is contained in $D$. We obtain

Proposition 1.3. Let $\mathscr{F}$ be a function space satisfying Condition A. Then $D_{\mathscr{F}}$ is involutive if and only if $\mathscr{F}$ is a function group.

Proof. Recall the basic identity concerning Lie brackets and Poisson brackets (cf. Abraham \& Marsden (1978)): for every $F, G: M \rightarrow \mathbb{R}$

$$
\left[X_{F}, X_{G}\right]=X_{\{F, G\}}
$$

Let $\mathscr{F}$ be a function group and $F_{1}, F_{2} \in \mathscr{F}$. Then $\left[X_{F_{1}}, X_{F_{2}}\right]=X_{\left\{F_{1}, F_{2}\right\}} \in D_{\mathscr{F}}$. Since $D_{\mathscr{F}}$ is spanned by Hamiltonian vectorfields this implies that $D_{\mathscr{F}}$ is involutive. Conversely, assume that $D_{\mathscr{F}}$ is involutive, and let $F_{1}, F_{2} \in \mathscr{F}$. Then $X_{\left\{F_{1}, F_{2}\right\}}=\left[X_{F_{1}}, X_{F_{2}}\right] \in D_{\mathscr{F}}$, and so by Lemma $1.2\left\{F_{1}, F_{2}\right\} \in \mathscr{F}$. Hence $\mathscr{F}$ is a function group.

Now we turn attention to the inclusions (1.10) and (1.11).

Proposition 1.4. Let $\mathscr{F}$ be a function group satisfying Condition A. Then

$$
\mathscr{F}=\left(\mathscr{F}^{\perp}\right)^{\perp}
$$


Furthermore, let $\mathscr{F}_{1}, \mathscr{F}_{2}$ be function groups satisfying Condition A. Assume $\mathscr{F}_{1}+\mathscr{F}_{2}$ is also a function group satisfying Condition A. Then

$$
\mathscr{F}_{1}+\mathscr{F}_{2}=\left(\mathscr{F}_{1}{ }^{\perp} \cap \mathscr{F}_{2}{ }^{\perp}\right)^{\perp}
$$

Assume $\mathscr{F}_{1}{ }^{\perp}+\mathscr{F}_{2}{ }^{\perp}$ is a function group satisfying Condition A. Then

$$
\mathscr{F}_{1} \cap \mathscr{F}_{2}=\left(\mathscr{F}_{1}{ }^{\perp}+\mathscr{F}_{2}{ }^{\perp}\right)^{\perp}
$$

Proof. (see also Weinstein (1983), Prop. 7.1). By Condition A, $\mathscr{F}=$ span $\left\{F_{1}, \ldots, F_{k}\right\}$, with $F_{i}$ independent. Let $F=\left(F_{1}, \ldots, F_{k}\right): M \rightarrow \mathbb{R}^{k}$, and denote the foliation of $M$ with leaves $F^{-1}(c), c \in \mathbb{R}^{k}$, by $\Phi$. By Prop. 1.4. $D_{g}$ is involutive and has constant dimension. Hence $D_{F}$ integrates to a foliation of $M$ which we denote by $\Phi^{\perp}$. It is easy to see that $\mathscr{F} \perp$ are precisely the functions which are constant along the leaves of $\Phi^{\perp}$ (Notice that $\mathscr{F}^{\perp}$ satisfies Condition $\mathrm{A}^{\prime}$, but not necessarily Condition A). Moreover $D_{\mathscr{F}}$ is the orthogonal complement of $D_{\mathscr{F}}$ under the symplectic structure $\omega$. Hence $\left(\mathscr{F}^{\perp}\right)^{\perp}=\mathscr{F}$.

For the proof of $(1.20)$ we denote the foliations corresponding to $\mathscr{F}_{1}$ and $\mathscr{F}_{2}$ by $\Phi_{1}$ and $\Phi_{2}$, and to $\mathscr{F}_{1}+\mathscr{F}_{2}$ by $\Phi_{1+2}$. By the first part of the proof we get foliations $\Phi_{1}^{\perp}, \Phi_{2}{ }^{\perp}$ and $\Phi_{1+2}^{\perp}$. It is clear that the leaves of $\Phi_{1+2}^{\perp}$ are exactly intersections of leaves of $\Phi_{1}^{\perp}$ and $\Phi_{2}^{\perp}$. By similar reasoning as in the first part one concludes to (1.21). For (1.22) we notice that by $(1.21), \mathscr{F}_{1} \perp+\mathscr{F}_{2} \perp=$ $\left(\left(\mathscr{F}_{1}{ }^{\perp}\right)^{\perp} \cap\left(\mathscr{F}_{2}{ }^{\perp}\right)^{\perp}\right)^{\perp}$, and so by $(1.20), \mathscr{F}_{1}{ }^{\perp}+\mathscr{F}_{2}{ }^{\perp}=\left(\mathscr{F}_{1} \cap \mathscr{F}_{2}\right)^{\perp}$, and $\mathscr{F}_{1} \cap$ $\mathscr{F}_{2}=\left(\mathscr{F}_{1}^{\perp}+\mathscr{F}_{2}^{\perp}\right)^{\perp}$

In Proposition 1.2 we saw that to every function group $\mathscr{F}$ satisfying Condition A there corresponds an involutive distribution $D_{\mathscr{F}}$ of constant dimension. The converse question is answered in

Proposition 1.5. Let $D$ be an involutive distribution of constant dimension. By Frobenius' theorem there exist locally independent functions $K_{1}, \ldots, K_{k}$ such that $D(x)=\operatorname{Kerspan}_{\mathbf{R}}\left\{d K_{1}(x), \ldots, d K_{k}(x)\right\}$. Assume that the functions $K_{1}, \ldots, K_{k}$ are globally defined. Then:

There exists a function group $\mathscr{F}$ such that $D=D_{\mathscr{F}}$ if and only if $\operatorname{span}\left\{K_{1}, \ldots, K_{k}\right\}$ is a function group. Moreover if $\operatorname{span}\left\{K_{1}, \ldots, K_{k}\right\}$ is a function group, then $\mathscr{F}=\left(\operatorname{span}\left\{K_{1}, \ldots, K_{k}\right\}\right)^{\perp}$.

Proof. Let $D=D_{\mathscr{F}}$, with $\mathscr{F}$ a function group. Then for any $F \in \mathscr{F}$

$$
\left\{K_{i}, F\right\}=-X_{F}\left(K_{i}\right)=-d K_{i}\left(X_{F}\right)=0, i=1, \ldots, k
$$

Jacobi's identity then implies

$$
\left\{\left\{K_{i}, K_{j}\right\}, F\right\}=d\left\{K_{i}, K_{j}\right\}\left(X_{F}\right)=0, \forall F \in \mathscr{F}, i, j=1, \ldots, k
$$

Hence $d\left\{K_{i}, K_{j}\right\}(x) \in \operatorname{span}_{\mathbf{R}}\left\{d K_{1}(x), \ldots, d K_{k}(x)\right\}, \forall x$. 
By Lemma 1.2 it follows that $\left\{K_{i}, K_{j}\right\} \in \operatorname{span}\left\{K_{1}, \ldots, K_{k}\right\}$. Hence $\operatorname{span}\left\{K_{1}, \ldots, K_{k}\right\}$ is a function group (even satisfying Condition A). It is clear that $\operatorname{span}\left\{K_{1}, \ldots, K_{k}\right\} \subset \mathscr{F} \perp$. Now let $G \in \mathscr{F}^{\perp}$. Then $\{G, F\}=-d G\left(X_{F}\right)=0$, $\forall F \in \mathscr{F}$, and hence $\operatorname{Ker} \operatorname{span}\left\{d K_{1}, \ldots, d K_{k}\right\}(x)=D_{\mathscr{F}}(x) \subset \operatorname{Ker} d G(x)$, or equivalently, $d G \in \operatorname{span}\left\{d K_{1}, \ldots, d K_{k}\right\}$. Therefore by Lemma $1.2, G \in$ $\operatorname{span}\left\{K_{1}, \ldots, K_{k}\right\}$. Hence $\operatorname{span}\left\{K_{1}, \ldots, K_{k}\right\}=\mathscr{F}^{\perp}$. Conversely, suppose that $\operatorname{span}\left\{K_{1}, \ldots, K_{k}\right\}$ is a function group. Since $D=\operatorname{Ker} \operatorname{span}\left\{d K_{1}, \ldots, d K_{k}\right\}, \operatorname{dim} D$ $=2 n-k$. Define $\mathscr{F}=\left(\operatorname{span}\left\{K_{1}, \ldots, K_{k}\right\}\right)^{\perp}$. Since $\operatorname{span}\left\{K_{1}, \ldots, K_{k}\right\}$ is a function group satisfying condition A it follows from Proposition 1.4 that $\operatorname{dim} d F=$ $2 n-k$. Furthermore it is clear that $D_{\mathscr{F}} \subset \operatorname{Ker} \operatorname{span}\left\{d K_{1}, \ldots, d K_{k}\right\}=D$. Since $\operatorname{dim} D_{\mathscr{F}}=\operatorname{dim} d \mathscr{F}=2 n-k$, necessarily $D=D_{\mathscr{F}}$.

\section{Controlled Invariance}

Consider an arbitrary affine nonlinear system

$$
\dot{x}=A(x)+\sum_{j=1}^{m} u_{j} B_{j}(x)
$$

with $A, B_{1}, \ldots, B_{m}$ smooth vectorfields. Define the distribution $\mathscr{B}:=$ $\operatorname{span}\left\{B_{1}, \ldots, B_{m}\right\}$ by $\mathscr{B}(x)=\operatorname{span}\left\{B_{1}(x), \ldots, B_{m}(x)\right\}$, and define the sum $D_{1}+$ $D_{2}$ of two distributions $D_{1}$ and $D_{2}$ as the smallest distribution containing $D_{1}$ as well as $D_{2}$.

An involutive distribution $D$ is invariant for (2.1) if
i) $[A, X] \subset D$
for every $X \in D, \quad j=1, \ldots, m$
ii) $\left[B_{j}, X\right] \subset D$

and locally controlled invariant (1.c.i.) if

i) $[A, X] \in D+\mathscr{B}$

ii) $\left[B_{j}, X\right] \in D+\mathscr{B}$ for every $X \in D, j=1, \ldots, m$

or more succinctly

i) $[A, D] \subset D+\mathscr{B}$

ii) $[\mathscr{B}, D] \subset D+\mathscr{B}$

Usually the following standard assumption is made (see Section 3)

Assumption 2. The distributions $D, \mathscr{B}$ and $D+\mathscr{B}$ all have constant dimension.

Roughly speaking, for a definition of local controlled invariance for Hamiltonian systems, we replace involutive distributions by function groups and Lie 
brackets by Poisson brackets. First we will state the definitions and develop the theory without making regularity assumptions as in Assumption 2.

Definition 2.1. Consider a Hamiltonian system (1.4) on $(M, \omega)$, and let $\mathscr{F}$ be a function group on $M$. Then $\mathscr{F}$ is invariant for (1.4) if
i) $\{H, F\} \subset \mathscr{F}$
for every $F \in \mathscr{F}, j=1, \ldots, m$
ii) $\left\{C_{j}, F\right\} \subset \mathscr{F}$

and locally controlled invariant if
i) $\{H, F\} \subset \mathscr{F}+\operatorname{span}\left\{C_{1}, \ldots, C_{m}\right\}$
for every $F \in \mathscr{F}, j=1, \ldots, m$,
ii) $\quad\left\{C_{j}, F\right\} \subset \mathscr{F}+\operatorname{span}\left\{C_{1}, \ldots, C_{m}\right\}$

or more succinctly if we define $\mathscr{C}:=\operatorname{span}\left\{C_{1}, \ldots, C_{m}\right\}$

$$
\begin{aligned}
& \{H, \mathscr{F}\} \subset \mathscr{F}+\mathscr{C} \\
& \{\mathscr{C}, \mathscr{F}\} \subset \mathscr{F}+\mathscr{C}
\end{aligned}
$$

Remark. In the above definition one can replace function groups by function spaces, just like one can take arbitrary distributions in (2.2). However if a function space $\mathscr{F}$ satisfies (2.3), then so does the function group $\bar{F}$. In fact let $F_{1}, F_{2} \in \mathscr{F}$. Then by the Jacobi-identity

$$
\begin{aligned}
\left\{H,\left\{F_{1}, F_{2}\right\}\right\} & =-\left\{F_{1},\left\{F_{2}, H\right\}\right\}-\left\{F_{2},\left\{H, F_{1}\right\}\right\} \in\left\{F_{1}, \mathscr{F}+\mathscr{C}\right\} \\
+ & \left\{F_{2}, \mathscr{F}+\mathscr{C}\right\} \subset \overline{\mathscr{F}}+\mathscr{C}, \text { and similarly }\left\{\mathscr{C},\left\{F_{1}, F_{2}\right\}\right\} \subset \overline{\mathscr{F}}+\mathscr{C} .
\end{aligned}
$$

The above definition is justified by the fact that a function group $\mathscr{F}$ is invariant, resp. locally controlled invariant, if and only if its corresponding distribution $D_{\mathscr{F}}$ is invariant, resp. locally controlled invariant:

Proposition 2.2. Let $\mathscr{F}$ be a function group satisfying Condition A. Then $\mathscr{F}$ is an invariant function group if and only if $D_{\mathscr{F}}$ is an invariant distribution. Moreover assume that $\mathscr{F}+\mathscr{C}$ satisfies Condition A. Then $\mathscr{F}$ is a l.c.i. function group if and only if $D_{F}$ is a l.c.i. distribution.

Proof. Let $\{H, F\} \subset \mathscr{F},\left\{C_{i}, F\right\} \subset F$ for any $\vec{F} \in \mathscr{F}$. Then $\left[X_{H}, X_{F}\right]=X_{\{H, F\}}$ $\in D_{\mathscr{F}}$ and $\left[X_{C_{i}}, X_{F}\right] \in D_{\mathscr{F}}$. So $D_{F}$ is invariant. Conversely if $\left[X_{H}, X_{F}\right]=X_{\{H, F\}} \in$ $D_{\mathscr{F}}$ then by Lemma $1.2,\{H, F\} \in \mathscr{F}$. Similarly for $\left[X_{C_{i}}, X_{F}\right]$. So $\mathscr{F}$ is invariant. Analogously if $\mathscr{F}$ is 1.c.i. then for any $F \in \mathscr{F},\left[X_{H}, X_{F}\right] \in D_{\mathscr{F}+\mathscr{G}}=D_{\mathscr{F}}+D_{\mathscr{G}}$ and $\left[X_{C_{i}, X_{F}}\right] \in D_{\mathscr{F}}+D_{\mathscr{G}}$. Therefore $D_{\mathscr{F}}$ is 1.c.i. (In this case $\mathscr{B}=D_{\mathscr{C}}$ ). Conversely if $\left[X_{H}, X_{F}\right]=X_{\{H, F\}} \in D_{\mathscr{F}}+D_{\mathscr{G}}$, then by Lemma 1.2, $\{H, F\} \in \mathscr{F}+\mathscr{C}$. Similarly $\left\{C_{i}, F\right\} \in \mathscr{F}+\mathscr{C}$. 
So every 1.c.i. function group $\mathscr{F}$ generates a l.c.i. involutive distribution $D_{\mathscr{F}}$. Conversely if $D=\operatorname{Ker}\left\{d K_{1}, \ldots, d K_{k}\right\}$ is a 1.c.i. involutive $(2 n-k)$-dimensional distribution, then by Proposition $1.5 \mathscr{F}=\left(\operatorname{span}\left\{K_{1}, \ldots, K_{k}\right\}\right)^{\perp}$ is a 1.c.i. function group if $\operatorname{span}\left\{K_{1}, \ldots, K_{k}\right\}$ is a function group. (Under the assumption that $\mathscr{F}+\mathscr{C}$ satisfies Condition A).

We now set up an algorithm, called the $\mathscr{F}^{*}$-algorithm, to produce the maximal 1.c.i. function group contained in a given function group. This algorithm is completely similar to the $\mathscr{V}^{*}$-algorithm in the linear case (Wonham (1979)), and the corresponding algorithm in the nonlinear case (cf. Isidori et al. (1981a)). We notice that the existence of a maximal 1.c.i. function group contained in a given function group $\mathscr{L}$ is already ensured by the following reasoning. Let $\mathscr{F}_{1}, \mathscr{F}_{2}$ be 1.c.i. and contained in $\mathscr{L}$. Then by an easy application of the Jacobi-identity $\overline{\mathscr{F}_{1}+\mathscr{F}_{2}}$ is again l.c.i. and contained in $\mathscr{L}$. Introducing a partial ordering on function groups by setting $\mathscr{F}_{1} \leqslant \mathscr{F}_{2}$ if $d \mathscr{F}_{1}(x) \subset d \mathscr{F}_{2}(x), \forall x \in M$, and applying Zorn's lemma, this implies that there exists a maximal l.c.i. function group $\mathscr{F}^{*}$ contained in $\mathscr{L}$.

$\mathscr{F} *$-algorithm. Define

$$
\begin{aligned}
& \mathscr{F}^{1}=\mathscr{L} \\
& \mathscr{F}^{i+1}=\mathscr{L} \cap(H+\mathscr{C})^{-1}\left(\mathscr{F}^{i}+\mathscr{C}\right) \quad i=1,2, \ldots
\end{aligned}
$$

Here $\mathscr{F}^{i}+\mathscr{C}$ denotes the smallest function space containing $\mathscr{C}$ as well as $\mathscr{F}^{i}$, and for an arbitrary function space $\mathscr{F}$,

$$
(H+\mathscr{C})^{-1}(\mathscr{F}):=\left\{G \in C^{\infty}(M) \mid\{H, G\} \subset \mathscr{F} \text { and }\{\mathscr{C}, G\} \subset \mathscr{F}\right\}
$$

Proposition 2.3. Let $\mathscr{F}^{i}$ be defined by (2.4). Then

a. $\mathscr{F}^{i+1} \subset \mathscr{F}^{i}, i=1,2, \ldots$

b. $\mathscr{F}^{i}$ is a function group, $i=1,2, \ldots$

c. If for a certain $k \mathscr{F}^{k+1}=\mathscr{F}^{k}$, then $\mathscr{F}^{k+1}=\mathscr{F}^{k}, \forall l$

Proof. a. By induction. It is clear that $\mathscr{F}^{2} \subset \mathscr{F}^{1}$. Now assume $\mathscr{F}^{i} \subset \mathscr{F}^{i-1}$. Let $F \in \mathscr{F}^{i+1}$. Then $F \in \mathscr{L}$ and $\{H, F\} \subset \mathscr{F}^{i}+\mathscr{C} \subset \mathscr{F}^{i-1}+\mathscr{C}$, as well as $\{\mathscr{C}, F\}$ $\subset \mathscr{F}^{i}+\mathscr{C} \subset \mathscr{F}^{i-1}+\mathscr{C}$. Hence $F \in \mathscr{F}^{i}$.

b. By induction. By definition $\mathscr{F}^{1}=\mathscr{L}$ is a function group. Let $\mathscr{F}^{i}$ be a function group, and take $F_{1}, F_{2} \in \mathscr{F}^{i+1}$.

Then

$$
\begin{aligned}
\left\{H,\left\{F_{1}, F_{2}\right\}\right\} & =-\left\{F_{1},\left\{F_{2}, H\right\}\right\}-\left\{F_{2},\left\{H, F_{1}\right\}\right\} \\
& \in\left\{F_{1}, \mathscr{F}^{i}+\mathscr{C}\right\}+\left\{F_{2}, \mathscr{F}^{i}+\mathscr{C}\right\} \subset \mathscr{F}^{i}+\mathscr{C},
\end{aligned}
$$

since $F_{1}, F_{2} \in \mathscr{F}^{i+1} \subset \mathscr{F}^{i}$ and $\mathscr{F}^{i}$ is a function group. 
Analogously $\left\{\mathscr{C},\left\{F_{1}, F_{2}\right\}\right\} \subset \mathscr{F}^{i}+C$. Hence $\mathscr{F}^{i}$ is closed under Poisson bracket. Now take a smooth function $G: \mathbb{R}^{2} \rightarrow \mathbb{R}$. Then

$$
\begin{aligned}
\left\{H, G\left(F_{1}, F_{2}\right)\right\} & =\frac{\partial G}{\partial x_{1}}\left(F_{1}, F_{2}\right)\left\{H, F_{1}\right\}+\frac{\partial G}{\partial x_{2}}\left(F_{1}, F_{2}\right)\left\{H, F_{2}\right\} \\
& \subset \mathscr{F}^{i+1} . \mathscr{F}^{i} \subset \mathscr{F}^{i},
\end{aligned}
$$

since $\frac{\partial G}{\partial x_{i}}\left(F_{1}, F_{2}\right) \in \mathscr{F}^{i+1} \subset \mathscr{F}^{i}$.

Analogously, $\left\{\mathscr{C}, G\left(F_{1}, F_{2}\right)\right\} \subset \mathscr{F}^{i}$. Hence $\mathscr{F}^{i}$ is a function group.

c. Immediate.

If there exists a $k$ such that $\mathscr{F}^{k+1}=\mathscr{F}^{k}$ then by $c$ the algorithm ends in $k$ steps and we denote $\mathscr{F}^{*}:=\mathscr{F}^{k}$. It follows that $\left\{H, \mathscr{F}^{*}\right\} \subset \mathscr{F}^{*}+\mathscr{C},\left\{\mathscr{C}, \mathscr{F}^{*}\right\} \subset$ $\mathscr{F}^{*}+\mathscr{C}$, so $\mathscr{F}^{*}$ is a l.c.i. function group contained in $\mathscr{L}$. Furthermore

Proposition 2.4. If the algorithm (2.4) ends in $k$ steps, then $\mathscr{F}^{*}=\mathscr{F}^{k}$ is the maximal locally controlled invariant function group contained in $\mathscr{L}$.

Proof. Let $\mathscr{F}$ be a l.c.i. function group contained in $\mathscr{L}$. By induction we will prove $\mathscr{F} \subset \mathscr{F}^{i}, i=1,2, \ldots$, and hence $\mathscr{F} \subset \mathscr{F}^{*}$. By assumption $\mathscr{F} \subset \mathscr{F}^{1}$. Suppose $\mathscr{F} \subset \mathscr{F}^{i}$. Then $\{H, \mathscr{F}\} \subset \mathscr{F}+\mathscr{C} \subset \mathscr{F}^{i}+\mathscr{C}$, and $\{\mathscr{C}, \mathscr{F}\} \subset \mathscr{F}+\mathscr{C} \subset \mathscr{F}^{i}+$ $\mathscr{C}$. Hence $F \subset F^{i+1}$.

Just as in the case of 1.c.i. distributions (see Assumption 2) we will make some regularity assumptions which will hold throughout the paper. First of all we assume that the 1.c.i. function groups $\mathscr{F}$ satisfy Condition A (Assumption 1). Furthermore in Definition 2.1 we make the following

Assumption 3. $C_{1}, \ldots, C_{m}$ are independent functions, so $\mathscr{C}$ is a function space satisfying Condition A. Furthermore $\mathscr{F}+\mathscr{C}$ satisfies Condition A.

With respec 8 to the $\mathscr{F}^{*}$-algorithm (2.4) we state

Assumption 4. The function groups $\mathscr{F}^{i}$ and the function spaces $\mathscr{F}^{i}+\mathscr{C}$ all satisfy Condition $A$.

Under Assumption 4 it is easy to conclude that the $\mathscr{F}^{*}$-algorithm ends in a finite number of steps, since $\operatorname{dim} \mathscr{F}^{i}(x)$ is a non-increasing function of $i$ and $\operatorname{dim} M$ is finite. (For this we may in fact replace Condition A by the weaker Condition $\mathrm{A}^{\prime}$.)

Let now $\mathscr{F}^{*}$ be the maximal 1.c.i. function group contained in $\mathscr{L}$. Then by Proposition 2.2 we know that $D_{\mathscr{F} *}$ is a 1.c.i. distribution contained in $\operatorname{Ker} d \mathscr{L}^{\perp}$. In general however, $D_{\mathscr{F} *}$ is not the maximal 1.c.i. distribution contained in 
$\operatorname{Ker} d \mathscr{L}^{\perp}$. This is only true if the maximal 1.c.i. distribution contained in $\operatorname{Ker} d \mathscr{L}^{\perp}$, denoted by $D^{*}$ is of the form $D_{\mathscr{F}}$ for a certain function group $\mathscr{F}$ (see Proposition 1.5.). In fact if $D^{*}=D_{\mathscr{F}}$, then $\mathscr{F}=\mathscr{F} *$. Furthermore notice that if we write $D^{*}=\operatorname{Ker} d \mathscr{K}$, then we always have $\mathscr{F}^{*} \subset \mathscr{K}^{\perp}$, since by Proposition 2.2 $D_{\mathscr{F} *}$ is a 1.c.i. distribution and so $D_{\mathscr{F} *} \subset D^{*} \subset \operatorname{ker} d \mathscr{K}$.

Example 2.5. Consider a Hamiltonian system on $\left(\mathbb{R}^{4}, d p_{1} \wedge d q_{1}+d p_{2} \wedge d q_{2}\right)$ with $H(q, p)=\frac{1}{2} e^{q_{2}} p_{1}^{2}+\frac{1}{2} p_{2}^{2}+\frac{1}{2} q_{1}^{2}+\frac{1}{2} q_{2}^{2}$ and $C(q, p)=q_{1}$. We want to compute the maximal 1.c.i. function group $\mathscr{F}^{*}$ contained in $\mathscr{C}^{\perp}=\operatorname{span}\left\{q_{1}, q_{2}, p_{2}\right\}$, and the maximal l.c.i. distribution $D^{*}$ contained in $\operatorname{Ker} d C=\operatorname{ker} d q_{1}$. Since $\{H, C\}=e^{q_{2}} p_{1}$, and $\{C,\{H, C\}\}=-e^{q_{2}}$ and so $X_{C}\left(X_{H}(C)\right) \neq 0$ it follows from Isidori et al. (1981a), that $D^{*}=\operatorname{Ker} \operatorname{span}\left\{d q_{1}, d\left(e^{q_{2}} p_{1}\right)\right\}$

In fact

$$
D^{*}=\operatorname{span}\left\{\frac{\partial}{\partial p_{2}}, p_{1} \frac{\partial}{\partial p_{1}}-\frac{\partial}{\partial q_{2}}\right\}
$$

Denote $\mathscr{K}=\operatorname{span}\left\{q_{1}, e^{q_{2}} p_{1}\right\}$, so $D^{*}=\operatorname{Ker} d \mathscr{K}$. Then $\mathscr{F}^{*} \subset \mathscr{K}^{\perp}$. Now by Proposition $1.4(\mathscr{K})^{\perp}=(\mathscr{\mathscr { K }})^{\perp}=\left(\operatorname{span}\left\{q_{1}, q_{2}, p_{1}\right\}\right)^{\perp}=\operatorname{span}\left\{q_{2}\right\}$. However $\left\{H, q_{2}\right\}=p_{2} \notin \operatorname{span}\left\{q_{2}\right\}+\operatorname{span}\left\{q_{1}\right\}$. Hence $\mathscr{K}^{\perp}$ is not a l.c.i. function group, and so $\mathscr{F}^{*}$ contains only the constant functions: $\mathscr{F}^{*}=\mathbb{R}$.

Remark. Local controlled invariance of function groups has an interesting global aspect. Let $\mathscr{F}$ be a l.c.i. function group satisfying Condition A. Then $\mathscr{F}$ defines a Poisson structure on $\mathbb{R}^{k}$, with $k=\operatorname{dim} d \mathscr{F}$. Now assume there exists a global basis $x_{1}, \ldots, x_{k}$ of $\mathbb{R}^{k}$ in which the Poisson structure is linear, i.e. the functions $w_{i j}$ take the form $w_{i j}(x)=\sum_{r=1}^{k} c_{i j r} x_{r}$. Then $\mathbb{R}^{k}$ can be interpreted as the dual $\mathfrak{g}^{*}$ of a Lie algebra $g$ with structure coefficients $c_{i j r}$ (see Weinstein (1983)). Denote the corresponding Lie group by $G$, then it follows that $D_{\mathscr{F}}$ is generated by a symplectic action of $G$ on $M$, and that the induced map $G \rightarrow \mathbb{R}^{k}$ is the momentum mapping of this action. In Nijmeijer \& van der Schaft (1984a) such a 1.c.i. distribution generated by a group action is called a partial symmetry.

\section{Hamiltonian Feedback}

As is well-known (Isidori et al. (1981b), Nijmeijer (1981)) the conditions of local controlled invariance of a distribution as stated in (2.2) are (under Assumption 2) equivalent to the local existence of a feedback which makes this distribution invariant (hence the name local controlled invariance). Precisely, let $D$ be an involutive distribution for a nonlinear system (2.1) such that Assumption 2 is satisfied. Then $D$ satisfies (2.2) if and only if there locally exists a feedback $u=\alpha(x)+\beta(x) v$, with $\alpha: M \rightarrow \mathbb{R}^{m}, \beta: M \rightarrow \mathbb{R}^{m \times m}$ smooth maps with $\operatorname{det} \beta(x)$ 
$\neq 0$, and $v=\left(v_{1}, \ldots, v_{m}\right)$ the new input vector, such that

$$
\begin{aligned}
& {\left[A+\sum_{j=1}^{m} \alpha_{j} B_{j}, D\right] \subset D} \\
& {\left[\sum_{j=1}^{m} \beta_{i j} B_{j}, D\right] \subset D \quad i=1, \ldots, m}
\end{aligned}
$$

I.e., $D$ is invariant for the feedback transformed system

$$
\dot{x}=A(x)+\sum_{j=1}^{m} \alpha_{j}(x) B_{j}(x)+\sum_{j=1}^{m} v_{j}\left(\sum_{i=1}^{m} \beta_{j i}(x) B_{i}(x)\right)
$$

Let now $\mathscr{F}$ be a l.c.i. function group for the Hamiltonian system (1.4) such that Assumptions 1 and 3 are satisfied. Then by Proposition $2.2 D_{F F}$ is a 1.c.i. distribution and hence there exists locally a feedback $u=\alpha(x)+\beta(x) v$ such that $D_{\mathscr{F}}$ is invariant for

$$
\dot{x}=X_{H}(x)-\sum_{j=1}^{m} \alpha_{j}(x) X_{C_{j}}(x)-\sum_{j=1}^{m} v_{j}\left(\sum_{i=1}^{m} \beta_{j i}(x) X_{C_{i}}(x)\right)
$$

However, in general the transformed system (3.3) is not Hamiltonian anymore.

Theorem 3.1. (van der Schaft $(1981,1983 b))$. Denote $C:=\left(C_{1}, \ldots, C_{m}\right): M \rightarrow Y$ for the Hamiltonian system (1.4). Then the system (3.3) is again Hamiltonian if and only if the feedback $u=\alpha(x)+\beta(x) v$ satisfies

i) There exists a function $P: Y \rightarrow \mathbb{R}$ such that $\sum_{j=1}^{m} \alpha_{j}(x) X_{C_{j}}(x)=X_{P \circ C}(x)$

ii) There exists a regular mapping $\left(R_{1}, \ldots, R_{m}\right): Y \rightarrow Y$ such that $\sum_{i=1}^{m} \beta_{j i}(x) X_{C_{j}}(x)=X_{R_{j} \circ c}(x) j=1, \ldots, m$

Such a feedback is called a Hamiltonian feedback $\left(P, R_{1}, \ldots, R_{m}\right)$. The feedback transformed system is the Hamiltonian system

$$
\begin{aligned}
& \dot{x}=X_{H-P \circ C}(x)-\sum_{j=1}^{m} v_{j} X_{R_{j} \circ C} \\
& y_{j}^{\prime}=R_{j} \circ C(x)
\end{aligned}
$$

Remark. Hamiltonian feedback has a direct physical interpretation. Part i) corresponds to the addition of a static Hamiltonian compensator

$$
u_{j}=\frac{\partial P}{\partial y_{j}}(y) \quad j=1, \ldots, m
$$


or said otherwise, the addition of an extra "potential energy" $P \circ C(x)$. In Part ii) we transform the outputs via the (coordinate) transformation $R:=\left(R_{1}, \ldots, R_{m}\right)$ : $Y \rightarrow Y$ and the inputs in a corresponding way via

$$
\left(v_{1}, \ldots, v_{m}\right)=\left(u_{1}, \ldots, u_{m}\right)\left(\frac{\partial R_{i}}{\partial y_{j}}(y)\right)^{-1}
$$

(The total induced mapping from $T^{*} Y$ to itself is a canonical transformation.) Pictorially

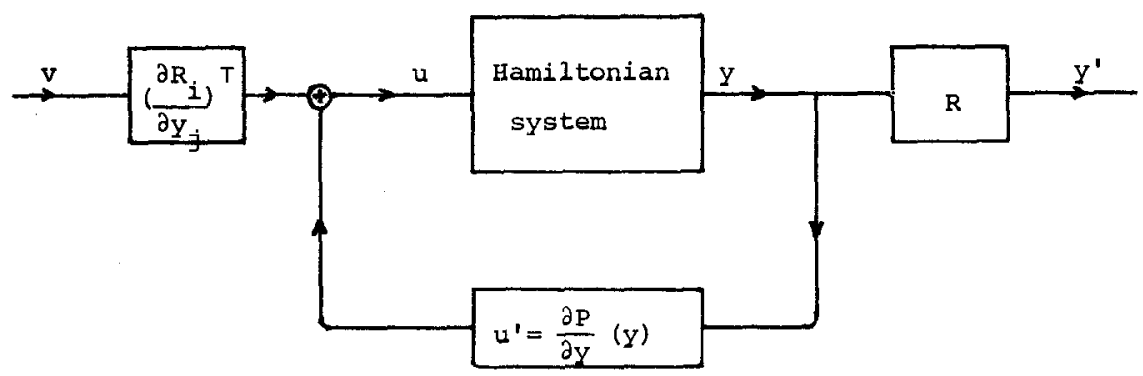

Furthermore Hamiltonian feedback is necessarily output feedback.

If there exists a Hamiltonian feedback $P, R_{1}, \ldots, R_{m}$ ) that makes $D_{\mathscr{F}}$ invariant then

$$
\begin{aligned}
& {\left[X_{H-P \circ C}, D_{\mathscr{F}}\right] \subset D_{\mathscr{F}}} \\
& {\left[X_{R_{j} \circ C}, D_{\mathscr{F}}\right] \subset D_{\mathscr{F}} \quad j=1, \ldots, m}
\end{aligned}
$$

or equivalently since $\mathscr{F}$ satisfies Condition A (see Prop. 2.2) $\{H-P \circ C, \mathscr{F}\} \subset$ $\mathscr{F},\left\{R_{j} \circ C, \mathscr{F}\right\} \subset \mathscr{F}, j=1, \ldots, m$. Hence $\mathscr{F}$ is invariant w.r.t. the feedback transformed Hamiltonian system.

Definition 3.2. A 1.c.i. function group $\mathscr{F}$ is called (locally) Hamiltonian controlled invariant ((1.)h.c.i.) if there exists (locally on $Y$ ) a Hamiltonian feedback $\left(P, R_{1}, \ldots, R_{m}\right)$ such that

$$
\begin{aligned}
& \{H-P \circ C, \mathscr{F}\} \subset \mathscr{F} \\
& \left\{R_{j} \circ C, \mathscr{F}\right\} \subset \mathscr{F} \quad j=1, \ldots, m .
\end{aligned}
$$

The central problem of this paper is now the following. Given a 1.c.i. function group $\mathscr{F}$, what additional conditions does $\mathscr{F}$ have to satisfy in order that $\mathscr{F}$ is locally Hamiltonian controlled invariant (1.h.c.i.). One additional condition is that $\mathscr{F}$ also has to be conditioned invariant, a concept which is treated in the next section. 


\section{Conditioned Invariance}

In this section we define conditioned invariance for Hamiltonian systems and treat its duality with controlled invariance.

Definition 4.1. Let (1.4) be a Hamiltonian system, and let $\mathscr{S}$ be a function group on $M$. Then $\mathscr{S}$ is conditioned invariant if

$$
\left\{H, \mathscr{S} \cap \mathscr{C}^{\perp}\right\} \subset \mathscr{S}
$$

The relation of Definition 4.1. with the usual definition of a conditioned invariant (or " $(h, f)$ "-invariant) distribution is the following. An involutive distribution $D$ for a general nonlinear system (2.1) is conditioned invariant if (cf. Isidori et al. (1981a), Nijmeijer \& van der Schaft (1982a))

$$
\begin{aligned}
& {[A, D \cap \operatorname{ker} d \mathscr{C}] \subset D} \\
& {\left[B_{j}, D \cap \operatorname{ker} d \mathscr{C}\right] \subset D \quad j=1, \ldots, m}
\end{aligned}
$$

In the case of a Hamiltonian system $A=X_{H}$ and $B_{j}=X_{C_{j}}, j=1, \ldots, m$. Now let $D$ satisfying (4.2) for a Hamiltonian system be generated by a function group $\mathscr{S}$, thus $D=D_{\mathscr{S}}$. Then (4.2.a) yields $X_{\left\{H, \mathscr{S} \cap \mathscr{Q}^{\perp}\right\}}=\left[X_{H}, D_{\mathscr{S}} \cap D_{\mathscr{G}}\right] \subset$ $\left[X_{H}, D_{\mathscr{S}} \cap \operatorname{ker} d \mathscr{C}\right] \subset D_{\mathscr{S}}$, since $D_{\mathscr{C} \perp} \subset \operatorname{ker} d \mathscr{C}$. So by Lemma $1.2\left\{H, \mathscr{S} \cap \mathscr{C}{ }^{\perp}\right\}$ $\subset \mathscr{S}$ (if $\mathscr{S}$ satisfies Condition A). Therefore, $\mathscr{S}$ is conditioned invariant. Moreover if $\mathscr{C}$ is a function group satisfying Condition $\mathrm{A}$ then by Proposition 1.5 ker $d \mathscr{C}=D_{\mathscr{G} \perp}$ and it is easy to see that (4.1) is actually equivalent to (4.2.a) Furthermore in this case (4.2.b) is automatically satisfied since $\left[X_{C_{j}}, D_{\mathscr{S}} \cap D_{\mathscr{C} \perp}\right]$ $\subset\left[X_{C_{j}}, D_{\mathscr{C}^{\perp}}\right]=0$. Therefore in general the notion of a conditioned invariant function group is slightly weaker than that of a conditioned invariant distribution, but if $\mathscr{C}$ is a function group the notions are equivalent (under the standard regularity assumptions).

We will now set up (completely similarly to the linear case) an algorithm to compute the minimal conditioned invariant function group containing a given function group, called the $\mathscr{S}^{*}$-algorithm. The existence of such a minimal function group is already ensured by the following argument. Let $\mathscr{S}^{1}, \mathscr{S}^{2}$ be conditioned invariant function groups containing a function group $\mathscr{N}$. Then $\mathscr{S}^{1} \cap \mathscr{S}^{2}$ is again a conditioned invariant function group containing $\mathscr{N}$. Hence by Zorn's lemma there exists a minimal one.

$\mathscr{S}^{*}$-algorithm. Define for a given function group $\mathscr{N}$

$$
\begin{aligned}
& \mathscr{S}^{1}=\mathscr{N} \\
& \mathscr{S}^{i+1}=\mathscr{N}+\left\{H, \overline{\mathscr{S}}^{i} \cap \mathscr{C}^{\perp}\right\} \quad i=1,2, \ldots
\end{aligned}
$$

where $\overline{\mathscr{S}}^{i}$ denotes the closure of $\mathscr{S}^{i}$ under Poisson bracket and $\mathscr{N}+$ $\left\{H, \overline{\mathscr{S}}^{i} \cap \mathscr{C}^{\perp}\right\}$ is the minimal function space containing $\mathscr{N}$ as well as $\left\{H, \overline{\mathscr{S}}^{i} \cap\right.$ $\left.\mathscr{C}^{\perp}\right\}$ (so by definition the $\mathscr{S}^{i}$ are function spaces). 
Proposition 4.2. Let $\mathscr{S}^{i}$ be defined by (4.3), then

a. $\mathscr{S}^{i} \subset \mathscr{S}^{i+1}, i=1,2, \ldots$

b. If for a certain $k, \mathscr{S}^{k}=\mathscr{S}^{k+1}$, then $\mathscr{S}^{k}=\mathscr{S}^{k+l}, \forall l$.

Proof. a. By induction. $\mathscr{S}^{1} \subset \mathscr{S}^{2}$ is clear. Assume $\mathscr{S}^{i-1} \subset \mathscr{S}^{i}$ and let $S^{i} \in \mathscr{S}^{i}$. Then there exists an $N \in \mathscr{N}$ and $S^{i-1} \in \overline{\mathscr{S}}^{i-1} \cap \mathscr{C}^{\perp}$ and a function $G: \mathbb{R}^{2} \rightarrow \mathbb{R}$ such that $S^{i}=G\left(N,\left\{H, S^{i-1}\right\}\right)$. Since by assumption $S^{i-1} \in \overline{\mathscr{S}}^{i-1} \subset \overline{\mathscr{S}}^{i}$, it follows that $S^{i} \in \mathscr{S}^{i+1}$.

b. Immediate.

If there exists a $k$ such that $\mathscr{S}^{k}=\mathscr{S}^{k+1}$ the algorithm ends in $k$ steps by part b., and we denote $\mathscr{S}^{*}=\overline{\mathscr{S}}^{k}$. It follows that $\left\{H, \overline{\mathscr{S}}^{k} \cap \mathscr{C}^{\perp}\right\} \subset \mathscr{S}^{k} \subset \overline{\mathscr{S}}^{k}$, and so $\mathscr{S}^{*}$ is a conditioned invariant function group containing $N$. Furthermore

Proposition 4.3. $\mathscr{S}^{*}$ is the minimal conditioned invariant function group containing $\mathcal{N}$.

Proof. Let $\mathscr{S}$ be a conditioned invariant function group with $\mathscr{N} \subset \mathscr{S}$. By induction we prove $\mathscr{S}^{i} \subset \mathscr{S}, \forall i$. Assume $\mathscr{S}^{i} \subset \overline{\mathscr{S}}^{i} \subset \mathscr{S}$. Then $\left\{H, \overline{\mathscr{S}}^{i} \cap C^{\perp}\right\} \subset$ $\left\{H, \mathscr{S} \cap \mathscr{C}^{\perp}\right\} \subset \mathscr{S}$, and so $\mathscr{S}^{i+1} \subset \mathscr{S}$.

Again we will make the regularity assumption that the function groups $\mathscr{S}$ in Definition 4 satisfy Condition A. With respect to the $\mathscr{S}^{*}$-algorithm we assume that the function groups $\overline{\mathscr{S}}^{i}$ all satisfy Condition $\mathrm{A}$. Then it is clear by dimensionality arguments that the $\mathscr{S}^{*}$-algorithm always ends in a finite number of steps.

We now consider the duality between (local) controlled invariance and conditioned invariance for Hamiltonian systems.

Proposition 4.4. Let $\mathscr{F}$ be a l.c.i. function group. Then $\mathscr{F} \perp$ is a conditioned invariant function group. Conversely let $\mathscr{S}$ be a conditioned invariant function group. Assume that $\mathscr{C}$ is a function group and that $\mathscr{S}^{\perp}+\mathscr{C}$ is a function group. Furthermore let $\mathscr{S}^{\perp}, \mathscr{S}^{\perp}, \mathscr{C}, \mathscr{S}^{\perp}+\mathscr{C}$ satisfy Condition A. Then $\mathscr{S}^{\perp}$ is a l.c.i. function group.

Proof. Let $\mathscr{F}$ be 1.c.i. Take an $F \in \mathscr{F}$ and an $F^{\perp} \in \mathscr{F} \perp \cap \mathscr{C} \perp$. Then $\left\{\left\{H, F^{\perp}\right\}, F\right\}=-\left\{\left\{F^{\perp}, F\right\}, H\right\}-\left\{\{F, H\}, F^{\perp}\right\} \in\left\{\mathscr{F}+\mathscr{C}, F^{\perp}\right\}=0$, since $F^{\perp} \in \mathscr{F}^{\perp} \cap \mathscr{C}^{\perp} \subset(\mathscr{F}+\mathscr{C})^{\perp}$ (see 1.11). Hence $\left\{H, \mathscr{F}^{\perp} \cap \mathscr{C}^{\perp}\right\} \subset \mathscr{F} \mathscr{F}^{\perp}$, and $\mathscr{F} \perp$ is conditioned invariant.

Conversely let $\mathscr{S}$ be conditioned invariant. Take an $S \in \mathscr{S} \cap \mathscr{C}^{\perp}$ and $S^{\perp} \in \mathscr{S}^{\perp}$. Then $\left\{\left\{H, S^{\perp}\right\}, S\right\}=-\left\{\left\{S^{\perp}, S\right\}, H\right\}-\left\{\{S, H\}, S^{\perp}\right\} \in\left\{\mathscr{S}, S^{\perp}\right\}$ $=0$. Hence $\left\{H, \mathscr{S}^{\perp}\right\} \subset\left(\mathscr{S}^{\prime} \cap \mathscr{C}^{\perp}\right)^{\perp}=\mathscr{S}^{\perp}+\mathscr{C}^{\perp \perp}=\mathscr{S}^{\perp}+\mathscr{C}$, by Proposition 1.4. Furthermore for any $C \in \mathscr{C},\left\{\left\{C, S^{\perp}\right\}, S\right\}=-\left\{\left\{S^{\perp}, S\right\}, C\right\}-\left\{\{S, C\}, S^{\perp}\right\}$ $=0$ and so $\left\{\mathscr{C}, \mathscr{S}^{\perp}\right\} \subset\left(\mathscr{S}^{\perp} \mathscr{C}^{\perp}\right)^{\perp}=\mathscr{S}^{\perp}+\mathscr{C}$.

Remark. We may also compare the $\mathscr{F}^{*}$-algorithm with the $\mathscr{S}^{*}$ algorithm. Let $\mathscr{L}^{\perp}=\mathscr{N}$ and define $\mathscr{F}^{i}$ and $\mathscr{S}^{i}$ according to (2.4), resp. (4.3). Then

a. $\mathscr{S}^{i} \subset \overline{\mathscr{S}}^{i} \subset\left(\mathscr{F}^{i}\right)^{\perp} \quad i=1,2, \ldots$

b. Assume that $\mathscr{C}$ and $\overline{\mathscr{S}}^{i}+\mathscr{C}, i=1,2, \ldots$ are function groups. Furthermore 
assume that $\overline{\mathscr{S}}^{i},\left(\overline{\mathscr{S}}^{i}\right)^{\perp}, \mathscr{C},\left(\overline{\mathscr{S}}^{i}\right)^{\perp}+\mathscr{C}$ all satisfy Condition A. Then $\overline{\mathscr{S}}^{i}=$ $\left(\mathscr{F}^{i}\right)^{\perp}, i=1,2, \ldots$, and so $\mathscr{S}^{*}=\left(\mathscr{F}^{*}\right)^{\perp}$.

Proof. a. By induction. Since $\mathscr{L}^{\perp}=\mathscr{N}, \mathscr{S}^{1}=\left(\mathscr{F}^{1}\right)^{\perp}$. Assume $\mathscr{S}^{i} \subset\left(\mathscr{F}^{i}\right)^{\perp}$. Take an $F \in \mathscr{F}^{i+1}$, i.e. $F \in \mathscr{L}^{\perp}$ and $\{H, F\} \in F^{i}+\mathscr{C},\{\mathscr{C}, F\} \subset \mathscr{F}^{i}+\mathscr{C}$. Then $\left\{\left\{H, \overline{\mathscr{S}}^{i} \cap \mathscr{C}\right\}, F\right\}=-\left\{\left\{\overline{\mathscr{S}}^{i} \cap \mathscr{C} \perp, F\right\}, H\right\}-\left\{\{F, H\}, \mathscr{\mathscr { S }}^{i} \cap \mathscr{C}^{\perp}\right\}$.

Now $F \in \mathscr{F}^{i+1} \subset \mathscr{F}^{i}$, and hence by assumption $\left\{F, \overline{\mathscr{S}}^{i}\right\}=0$. Furthermore

$$
\left\{\{H, F\}, \overline{\mathscr{S}}^{i} \cap \mathscr{C}^{\perp}\right\} \subset\left\{\mathscr{F}^{i}+\mathscr{C}, \overline{\mathscr{S}}^{i} \cap \mathscr{C}^{\perp}\right\}=0, \quad \text { since } \overline{\mathscr{S}}^{i} \subset\left(\mathscr{F}^{i}\right)^{\perp}
$$

Hence $\mathscr{S}^{i+1} \subset\left(\mathscr{F}^{i+1}\right)^{\perp}$.

b. By induction. Assume $\mathscr{F}^{i}=\left(\overline{\mathscr{S}}^{i}\right)^{\perp}$. Then $\overline{\mathscr{S}}^{i} \subset\left(\overline{\mathscr{S}}^{i}\right)^{\perp \perp}=\left(\mathscr{F}^{i}\right)^{\perp}$ and therefore by part a. $\overline{\mathscr{S}}^{i+1} \subset\left(\mathscr{F}^{i+1}\right)^{\perp}$. Now take an $S^{\perp} \in\left(\overline{\mathscr{S}}^{i+1}\right)^{\perp}$. Then $S^{\perp} \in \mathscr{L}^{\perp}$ and $S^{\perp} \in\left\{H, \overline{\mathscr{S}}^{i} \cap \mathscr{C}^{\perp}\right\}^{\perp}$. This last inclusion means that for any $S^{i} \in \overline{\mathscr{S}}^{i} \cap \mathscr{C}^{\perp},\left\{S^{\perp},\left\{H, S^{i}\right\}\right\}=0$. Now

$$
\left\{S^{\perp},\left\{H, S^{i}\right\}\right\}=-\left\{H,\left\{S^{i}, S^{\perp}\right\}\right\}-\left\{S^{i},\left\{S^{\perp}, H\right\}\right\}
$$

and since $\mathscr{S}^{i} \in \overline{\mathscr{S}}^{i} \subset \overline{\mathscr{S}}^{i+1}$ this implies that $\left\{\left\{H, S^{\perp}\right\}, S^{i}\right\}=0$ for any $S^{i} \in \overline{\mathscr{S}}^{i}$ $\cap \mathscr{C} \perp$.

Hence $\left\{H,\left(\overline{\mathscr{S}}^{i+1}\right)^{\perp}\right\} \subset\left(\overline{\mathscr{S}}^{i} \cap \mathscr{C}^{\perp}\right)^{\perp}=\left(\overline{\mathscr{S}}^{i}\right)^{\perp}+\mathscr{C} \mathscr{C}^{\perp}$, by Proposition 1.4. Analogously $\left\{\mathscr{C},\left(\overline{\mathscr{S}}^{i+1}\right)^{\perp}\right\} \subset\left(\overline{\mathscr{S}}^{i}\right)^{\perp}+\mathscr{C}$. By assumption $\left(\overline{\mathscr{S}}^{i}\right)^{\perp}=\mathscr{F}^{i}$ and hence $\left(\overline{\mathscr{S}}^{i+1}\right)^{\perp} \subset \mathscr{F}^{i+1}$. Together, for any $i$ we have $\mathscr{F}^{i}=\left(\mathscr{F}^{i}\right)^{\perp} \perp \subset\left(\overline{\mathscr{S}}^{i}\right)^{\perp} \subset$ $\mathscr{F}^{i}$, and hence $\left(\overline{\mathscr{S}}^{i}\right)^{\perp}=\mathscr{F}^{i}$.

Example 4.5. We continue Example 2.5 and calculate the minimal conditioned invariant function group containing $\mathscr{C}=\operatorname{span}\left\{q_{1}\right\}$.

$$
\begin{aligned}
& \mathscr{S}^{1}=\operatorname{span}\left\{q_{1}\right\} \\
& \mathscr{S}^{2}=\operatorname{span}\left\{q_{1}\right\}+\left\{H, \mathscr{C} \cap \mathscr{C}^{\perp}\right\}=\operatorname{span}\left\{q_{1}, e^{q_{2}} p_{1}\right\} \\
& \text { Hence } \overline{\mathscr{S}}^{2}=\operatorname{span}\left\{q_{1}, q_{2}, p_{1}\right\} \text { and so } \\
& \mathscr{S}^{3}=\operatorname{span}\left\{q_{1}\right\}+\operatorname{span}\left\{e^{q_{2}} p_{1}, p_{2}, q_{1}\right\} \text { and } \\
& \overline{\mathscr{S}}^{3}=\operatorname{span}\left\{q_{1}, q_{2}, p_{1}, p_{2}\right\}=C^{\infty}\left(\mathbb{R}^{4}\right) .
\end{aligned}
$$

Therefore $\mathscr{S}^{*}=\overline{\mathscr{S}}^{3}=C^{\infty}\left(\mathbb{R}^{4}\right)$ and $\mathscr{F}^{*}$ (see Example 2.5$)=\left(\mathscr{S}^{*}\right)^{\perp}=\mathbb{R}$, in agreement with the above remark.

\section{Invariance by Hamiltonian Feedback}

We return to the central problem of finding conditions in order that a function group $\mathscr{F}$ for a Hamiltonian system (1.4) is locally Hamiltonian controlled invariant, i.e., in order that there locally exist functions $P, R_{1}, \ldots, R_{m}$ on $Y$ such 
that

$$
\begin{aligned}
& \{H-P \circ C, \mathscr{F}\} \subset \mathscr{F} \\
& \left\{R_{j} \circ C, \mathscr{F}\right\} \subset \mathscr{F} \quad j=1, \ldots, m
\end{aligned}
$$

We noticed already that $\mathscr{F}$ has to be at least locally controlled invariant. Furthermore let $\mathscr{F}$ satisfy (5.1). Then

$$
\left\{H, \mathscr{F} \cap \mathscr{C}^{\perp}\right\}=\left\{H-P \circ C, \mathscr{F} \cap \mathscr{C}^{\perp}\right\} \subset \mathscr{F}
$$

Hence $\mathscr{F}$ also has to be conditioned invariant. Moreover if (5.1) is satisfied it follows from the Jacobi-identity that $\{H-P \circ C, \mathscr{F} \perp\} \subset \mathscr{F}^{\perp}$. Hence $\{H, \mathscr{F} \perp\}$ $\subset \mathscr{F}^{\perp}+\mathscr{C}$ and as in (5.2) $\left\{H, \mathscr{F}^{\perp} \cap \mathscr{C}^{\perp}\right\} \subset \mathscr{F}^{\perp}$. Similarly $\left\{R_{j} \circ C, \mathscr{F}^{\perp}\right\} \subset$ $\mathscr{F} \perp$ and so $\left\{C, F^{\perp}\right\} \subset \mathscr{F}^{\perp}+\mathscr{C}$. Therefore if $\mathscr{F}$ is 1.h.c.i. then $\mathscr{F}$ as well as $\mathscr{F} \perp$ have to be locally controlled and conditioned invariant. However in Proposition 4.4. we already derived that if $\mathscr{F}$ is 1.c.i. then $\mathscr{F} \perp$ is conditioned invariant, and hence if $\mathscr{F}^{\perp}$ is 1.c.i. then $\mathscr{F}=\left(\mathscr{F}^{\perp}\right)^{\perp}$ is conditioned invariant (see Prop. 1.4). So a necessary condition for $\mathscr{F}$ to be 1.h.c.i. is that $\mathscr{F}$ and $\mathscr{F} \perp$ are both locally controlled invariant.

One may suspect that this condition (maybe under some additional integrability and regularity conditions) is also sufficient for $\mathscr{F}$ to be locally Hamiltonian controlled invariant. However this is not true as already shown by the linear case (see van der Schaft (1983a), Nijmeijer \& van der Schaft (1984b)). In this case $\mathscr{F}$ is spanned by linear functions on $\mathbb{R}^{2 n}$ and $D_{\mathscr{F}}$ corresponds to a linear subspace $\mathscr{V}$ of $\mathbb{R}^{2 n}$. Moreover $\mathscr{F}$ is 1.c.i. if and only if $\mathscr{F}$ is conditioned invariant. So the condition that $\mathscr{F}$ and $\mathscr{F} \perp$ are 1.c.i. is equivalent to $\mathscr{F}$ being controlled and conditioned invariant. This implies that there exists output feedback $u=K y$ which makes $\mathscr{V}$ invariant. Now output feedback $u=K y$ is Hamiltonian feedback if and only if $K=K^{T}$. However in general $K$ cannot be taken to be symmetric. Only in case $\mathscr{F}$ is Lagrangian or symplectic this is always possible.

Definition 5.1. Let $\mathscr{F}$ be a function group. $\mathscr{F}$ is Lagrangian if $\mathscr{F} \perp=\mathscr{F}$ and symplectic if $\mathscr{F}^{\perp} \cap \mathscr{F}=\mathbb{R}$. Furthermore $\mathscr{F}$ is coisotropic if $\mathscr{F} \perp \subset \mathscr{F}$.

Remark. If $\mathscr{F}$ is coisotropic then $\operatorname{dim} d \mathscr{F}(x) \geqslant \frac{1}{2} \operatorname{dim} M$. Furthermore a coisotropic $\mathscr{F}$ is Lagrangian if and only if $\operatorname{dim} d \mathscr{F}(x)=\frac{1}{2} \operatorname{dim} M$.

Therefore also in the nonlinear case for arbitrary $\mathscr{F}$ the condition that $\mathscr{F}$ and $\mathscr{F} \perp$ are l.c.i. may only imply that $D_{\mathscr{F}}$ can be made invariant by output feedback, not necessarily Hamiltonian feedback. In fact in the nonlinear case there is an extra complication because controlled and conditioned invariance is not enough for the existence of output feedback. We need an extra integrability condition as shown in Nijmeijer \& van der Schaft (1982a).

In this section we shall show that also in the nonlinear case a Lagrangian function group $\mathscr{F}$ which is 1.c.i. (and hence also $\mathscr{F} \perp=\mathscr{F}$ is 1.c.i.) can be made invariant by Hamiltonian feedback, provided the extra integrability condition for the existence of output feedback is satisfied. Moreover for Hamiltonian systems this condition can be stated in a much more concrete way than for general 
nonlinear systems. Preliminary investigations (Nijmeijer \& van der Schaft (1984c)) suggest that also symplectic nonlinear functions groups $\mathscr{F}$ are 1.h.c.i. if $\mathscr{F}$ and $\mathscr{F} \perp$ are 1.c.i., again provided the extra integrability condition is satisfied. Furthermore in the next section we shall show how coisotropic 1.c.i. function groups may be made invariant by dynamic Hamiltonian feedback, by lifting the function group to a Lagrangian 1.c.i. function group for an augmented Hamiltonian system.

Remark 1. In all these three cases $(\mathscr{F}=\mathscr{F} \perp, \mathscr{F} \perp \subset \mathscr{F}, \mathscr{F} \cap \mathscr{F} \perp=\mathbb{R}) \mathscr{F}$ satisfies the conditions of Theorem 1.1, so we may derive a local normal form for $\mathscr{F}$.

Remark 2. We have argued that only under severe conditions a 1.c.i. function group $\mathscr{F}$ is also 1.h.c.i. So in general there only exists a non-Hamiltonian feedback $u=\alpha(x)+\beta(x) \quad v$ which makes $D_{\mathscr{F}}$ invariant. Although the resulting transformed system $\dot{x}=X_{H}-\sum_{j=1}^{m} \alpha_{j} X_{C_{j}}+\sum_{i=1}^{m} v_{i} \sum_{j=1}^{m} \beta_{i j} X_{C_{j}}$ is therefore not Hamiltonian it can be made again Hamiltonian by the addition of output injection, i.e.

$$
\begin{aligned}
\dot{x} & =X_{H}-\sum_{j=1}^{m} \alpha_{j} X_{C_{j}}-\sum_{j=1}^{m} C_{j} X_{\alpha_{j}}+\sum_{i=1}^{m} v_{i}\left(\sum_{i=1}^{m} \beta_{i j} X_{C_{j}}+\sum_{j=1}^{m} C_{j} X_{\beta_{i j}}\right) \\
& =X_{H}-\sum_{j=1}^{m} \alpha_{j} C_{j}+\sum_{i=1}^{m} v_{i} X_{\left(\sum_{j-1}^{m} \beta_{i j} C_{j}\right)}
\end{aligned}
$$

This is investigated in van der Schaft (1985), and gives a hint to handle 1.c.i. function groups which are not 1.h.c.i. in a "Hamiltonian way".

So let $\mathscr{F}$ be a 1.c.i. Lagrangian function group on $(M, \omega)$, satisfying Condition A. Then there exist $n$ independent functions $F_{1}, \ldots, F_{n}$ such that $\mathscr{F}=$ $\operatorname{span}\left\{F_{1}, \ldots, F_{n}\right\}$. As we saw in Section $1 F$ defines a Poisson structure on $\mathbb{R}^{n}$. In this case because $\mathscr{F}=\mathscr{F} \perp$ the Poisson structure is identically zero, i.e. $\left\{G_{1}, G_{2}\right\}_{\mathbf{R}} n=0, \forall G_{1}, G_{2}: \mathbb{R}^{n} \rightarrow \mathbb{R}$. However the following construction works for arbitrary $\mathscr{F}$. Define $F: M \rightarrow \mathbb{R}^{n}$ as $F=\left(F_{1}, \ldots, F_{n}\right)$ and let $z=\left(z_{1}, \ldots, z_{n}\right)$ be coordinates for $\mathbb{R}^{n}$ such that $z_{i} \circ F=F_{i}, i=1, \ldots, n$. Let furthermore $C:=$ $\left(C_{1}, \ldots, C_{m}\right): M \rightarrow Y$ and let $y=\left(y_{1}, \ldots, y_{m}\right)$ be (local) coordinates for $Y$ such that $y_{j} \circ C=C_{j}, j=1, \ldots, m$. Denote by $(y, u)=\left(y_{1}, \ldots, y_{m}, u_{1}, \ldots, u_{m}\right)$ the corresponding natural coordinates for $T^{*} Y . T^{*} Y$ has the canonically defined Poisson (in fact symplectic) structure

$$
\left\{G_{1}(y, u), G_{2}(y, u)\right\}_{T^{*} Y}=\sum_{j=1}^{m}\left(\frac{\partial G_{1}}{\partial u_{j}} \frac{\partial G_{2}}{\partial y_{j}}-\frac{\partial G_{1}}{\partial y_{j}} \frac{\partial G_{2}}{\partial u_{j}}\right)
$$

for $G_{1}, G_{2}: T^{*} Y \rightarrow \mathbb{R}$. Therefore we can give $T^{*} Y \times \mathbb{R}^{n}$ the product structure of the Poisson structures on $T^{*} Y$ respectively $\mathbb{R}^{n}$ :

$$
\begin{aligned}
& \left\{G_{1}(y, u, z), G_{2}(y, u, z)\right\}_{T^{*} Y \times \mathbf{R}^{n}} \\
& \quad=\left\{G_{1}(y, u, z), G_{2}(y, u, z)\right\}_{T^{*} Y}+\left\{G_{1}(y, u, z), G_{2}(y, u, z)\right\}_{\mathbf{R}^{n}} \\
& \quad=\left\{G_{1}(y, u, z), G_{2}(y, u, z)\right\}_{T^{*} Y}
\end{aligned}
$$


since the Poisson structure on $\mathbf{R}^{n}$ is zero. (Notice that for computing $\left\{G_{1}(y, u, z), G_{2}(y, u, z)\right\}_{T^{*} Y}$ we treat $z$ as a parameter). Since $\mathscr{F}$ is 1.c.i.

$$
\left\{H, F_{i}\right\} \subset \mathscr{F}+\mathscr{C},\left\{C_{j}, F_{i}\right\} \subset \mathscr{F}+\mathscr{C}
$$

and hence under Assumption 3 there exist smooth functions $K_{j}^{i}(y, z), V^{i}(y, z)$ such that

$$
\begin{array}{ll}
\left\{H, F_{i}\right\}(x)=V^{i}(C(x), F(x)) & i=1, \ldots, n \\
\left\{C_{j}, F_{i}\right\}(x)=-K_{j}^{i}(C(x), F(x)) & i=1, \ldots, n \\
j & =1, \ldots, m
\end{array}
$$

or equivalently

$$
\left\{H-\sum_{j=1}^{m} u_{j} C_{j}, F_{i}\right\}_{M}(x)=F_{i}^{e}(C(x), u, F(x)) \quad i=1, \ldots, n
$$

with $F_{i}^{e}: T^{*} Y \times \mathbb{R}^{n} \rightarrow \mathbb{R}$ defined by

$$
F_{i}^{e}(y, u, z)=V^{i}(y, z)+\sum_{j=1}^{m} u_{j} K_{j}^{i}(y, z) \quad i=1, \ldots, n
$$

We now state the main theorem.

Theorem 5.2. Let $(1.4)$ be a Hamiltonian system, and let $\mathscr{F}=\operatorname{span}\left\{F_{1}, \ldots, F_{n}\right\}$ be a Lagrangian function group on $M$. Suppose

1. $\mathscr{F}$ is l.c.i., so there exist functions $F_{1}^{e}, \ldots, F_{n}^{e}: T^{*} Y \times \mathbb{R}^{n} \rightarrow \mathbb{R}$ such that (5.7) holds.

Moreover suppose $\mathscr{F}$ satisfies the integrability condition:

2. The function space $\operatorname{span}\left\{F_{1}^{e}, \ldots, F_{n}^{e}\right\}$ on $T^{*} Y \times \mathbb{R}^{n}$ "projects" to a function space on $T^{*} Y$, i.e. there exist functions $G_{1}^{e}, \ldots, G_{n}^{e}: T^{*} Y \rightarrow \mathbb{R}$ such that

$$
\begin{aligned}
& \underset{i=1, \ldots, n}{\operatorname{span}}\left\{\sum_{j=1}^{m}\left(\frac{\partial F_{i}^{e}}{\partial y_{j}} d y_{j}+\frac{\partial F_{i}^{e}}{\partial u_{j}} d u_{j}\right)\right\} \\
& \quad=\operatorname{span}_{i=1, \ldots, n}\left\{\sum_{j=1}^{m}\left(\frac{\partial G_{i}^{e}}{\partial y_{j}} d y_{j}+\frac{\partial G_{i}^{e}}{\partial u_{j}} d u_{j}\right)\right\}=\operatorname{span}_{i=1, \ldots, n}\left\{d G_{i}^{e}\right\}
\end{aligned}
$$

in every point $(y, u, z)$ of $T^{*} Y \times \mathbb{R}^{n}$.

Furthermore suppose the following regularity assumptions are satisfied. 
3. The function spaces $\mathscr{F}, \mathscr{C}$ and $\mathscr{F}+\mathscr{C}$ satisfy Condition $\mathrm{A}$.

4. The function spaces $\mathscr{F}^{e}:=\operatorname{span}\left\{F_{1}^{e}, \ldots, F_{n}^{e}\right\}$ on $T^{*} Y \times \mathbb{R}^{n}$, and $\mathscr{G}^{e}:=$ $\operatorname{span}\left\{G_{1}^{e}, \ldots, G_{n}^{e}\right\}$ on $T^{*} Y$ satisfy Condition A.

5. The codistribution $\operatorname{span}_{i=1, \ldots, n}\left\{\frac{\partial F_{i}^{e}}{\partial u_{j}} d u_{j}\right\}$ on $T^{*} Y \times \mathbb{R}^{n}$ has constant dimension. Then there exist locally on $Y$ functions $P, R_{1}, \ldots, R_{m}$ with $\operatorname{det}\left(\frac{\partial R_{i}}{\partial y_{j}}\right) \neq 0$, such that (5.1) holds. Conversely, if (5.1) holds, then $\mathscr{F}$ satisfies 1 . and 2.

Remark. Since the theorem is essentially local in nature, we may replace Condition $\mathrm{A}$ by Condition $\mathrm{A}^{\prime}$.

Proof. Let $\mathscr{F}$ satisfy 1 up till 5. First we will prove that $\mathscr{F}^{e}$ as well as $\mathscr{G}^{e}$ are function groups, such that $\left\{F_{i}^{e}, F_{j}^{e}\right\}_{T^{*} Y \times \mathbf{R}^{n}}=\left\{G_{i}^{e}, G_{j}^{e}\right\}_{T^{*} Y}=0, i, j=1, \ldots, n$.

Consider $F_{1}, F_{2} \in \mathscr{F}$. By application of the Jacobi-identity in every $x=$ $(q, p) \in M$ (see also van der Schaft $(1983 \mathrm{c})$ )

$$
\begin{aligned}
\{H- & \left.\sum_{j=1}^{m} u_{j} C_{j},\left\{F_{1}, F_{2}\right\}\right\}(x) \\
= & \left\{\left\{H-\sum_{j=1}^{m} u_{j} C_{j}, F_{1}\right\}, F_{2}\right\}(x)-\left\{\left\{H-\sum_{j=1}^{m} u_{j} C_{j}, F_{2}\right\}, F_{1}\right\}(x) \\
= & \left\{F_{1}^{e}(C(x), u, F(x)), F_{2}(x)\right\}-\left\{F_{2}^{e}(C(x), u, F(x)), F_{1}(x)\right\} \\
= & \sum_{j=1}^{m} \frac{\partial F_{1}^{e}}{\partial y_{j}}\left\{C_{j}, F_{2}\right\}+\sum_{i=1}^{n} \frac{\partial F_{1}^{e}}{\partial z_{i}}\left\{F_{i}, F_{2}\right\} \\
& -\sum_{j=1}^{m} \frac{\partial F_{2}^{e}}{\partial y_{j}}\left\{C_{j}, F_{1}\right\}-\sum_{i=1}^{n} \frac{\partial F_{2}^{e}}{\partial z_{i}}\left\{F_{i}, F_{1}\right\} \\
= & \sum_{j=1}^{m} \frac{\partial F_{1}^{e}}{\partial y_{j}}\left\{C_{j}, F_{2}\right\}-\sum_{j=1}^{m} \frac{\partial F_{2}^{e}}{\partial y_{j}}\left\{C_{j}, F_{1}\right\},
\end{aligned}
$$

since $\mathscr{F}$ is Lagrangian.

Now $F_{i}^{e}=\sum_{j=1}^{m} u_{j} K_{j}^{i}(y, z)+V^{i}(y, z), i=1, \ldots, n$. Hence

$$
\frac{\partial F_{i}^{e}}{\partial y_{r}}=\sum_{j=1}^{m} u_{j} \frac{\partial K_{j}^{i}}{\partial y_{r}}+\frac{\partial V^{i}}{\partial y_{r}}
$$

and

$$
\left\{C_{j}, F_{i}\right\}=-K_{j}^{i}=-\frac{\partial F_{i}^{e}}{\partial u_{j}}
$$


Therefore

$$
\begin{aligned}
\{H & \left.-\sum_{j=1}^{m} u_{j} C_{j},\left\{F_{1}, F_{2}\right\}\right\}(x) \\
& =\sum_{j=1}^{m}\left(\frac{\partial F_{1}^{e}}{\partial u_{j}} \frac{\partial F_{2}^{e}}{\partial y_{j}}-\frac{\partial F_{1}^{e}}{\partial y_{j}} \frac{\partial F_{2}^{e}}{\partial u_{j}}\right)(C(x), u, F(x)) \\
& =\left\{F_{1}^{e}, F_{2}^{e}\right\}_{T^{*} Y \times \mathbf{R}^{n} .}
\end{aligned}
$$

Hence the mapping $F_{i} \rightarrow F_{i}^{e}$ from $\mathscr{F}$ to $\mathscr{F}^{e}$ is an algebra morphism with respect to the Poisson bracket on $M$, respectively on $T^{*} Y \times \mathbb{R}^{n}$. Therefore $\mathscr{F}^{e}$ is a function group, and since $\mathscr{F}$ is Lagrangian

$$
\left\{F_{i}^{e}, F_{j}^{e}\right\}_{T^{*} Y \times \mathbf{R}^{n}}=0 \text {, for any } F_{i}^{e}, F_{j}^{e} \in \mathscr{F}^{e} \text {. }
$$

Since the Poisson structure on $\mathbb{R}^{n}$ is zero it follows that also $\mathscr{G}^{e}$ is a function group with $\left\{G_{i}^{e}, G_{j}^{e}\right\}_{T^{*} Y}=0$.

Now turn attention to the functions $F_{i}^{e}$. By condition 5. the rank of the matrix

$$
\left(\begin{array}{ccc}
K_{1}^{1} & \cdots & K_{1}^{n} \\
\vdots & & \vdots \\
K_{m}^{1} & \cdots & K_{m}^{n}
\end{array}\right)
$$

is constant, say $r$, in every point $(y, z)$. Hence we may take $F_{1}, \ldots, F_{n}$ spanning $\mathscr{F}^{e}$ in such a way that $K_{1}^{i}(y, z)=\cdots=K_{m}^{i}(y, z)=0, \forall(y, z), i=r+1, \ldots, n$. Then, equivalently $\left\{C_{j}, F_{i}\right\}$ is identically zero, $j=1, \ldots, m, i=r+1, \ldots, n$, and hence $F_{i} \in \mathscr{C}^{\perp}, i=r+1, \ldots, n$. Since $\mathscr{F}$ is conditioned invariant this implies that $\left\{\boldsymbol{H}, \boldsymbol{F}_{i}\right\} \subset \mathscr{F}, i=\boldsymbol{r}+1, \ldots, n$. Hence $V^{i}$ can be taken as a function of $z$ only, $i=r+1, \ldots, n$. Since the $F_{i}^{e}$ are affine in $u$, the $G_{i}^{e}$ can be also taken affine in $u$, i.e.

$$
G_{i}^{e}(y, u)=\sum_{j=1}^{m} u_{j} L_{j}^{i}(y)+W^{i}(y) \quad i=1, \ldots, n
$$

Furthermore by the above reasoning we may take $k$ independent functions $G_{1}^{e}, \ldots, G_{k}^{e}$ of the form (5.11) and spanning $\mathscr{G}^{e}$ such that

$$
\operatorname{dimspan}\left\{\left(\begin{array}{c}
L_{1}^{1}(y) \\
\vdots \\
L_{m}^{1}(y)
\end{array}\right), \ldots,\left(\begin{array}{c}
L_{1}^{k}(y) \\
\vdots \\
L_{m}^{k}(y)
\end{array}\right)\right\}=k
$$

It follows that the Hamiltonian vectorfields $X_{G_{1}^{e}}, \ldots, X_{G_{k}^{e}}$ on $T^{*} Y$ are independent and project to $k$ independent vectorfields on $Y$. In fact, denote the projection from $T^{*} Y$ to $Y$ by $\pi$, then

$$
\pi_{*} X_{G_{i}^{e}}=\sum_{j=1}^{m} L_{j}^{i}(y) \frac{\partial}{\partial y_{j}} \quad i=1, \ldots, k
$$


Since $\left\{G_{i}^{e}, G_{j}^{e}\right\}_{T^{*} Y}=0$, it follows that

$$
\left[\pi_{*} X_{G_{i}^{e}}, \pi_{*} X_{G_{j}^{e}}\right]=0 \quad i, j=1, \ldots, k
$$

Hence there exists a local coordinate transformation $\left(R_{1}(y), \ldots, R_{m}(y)\right)=$ $\left(y_{1}^{\prime}, \ldots, y_{m}^{\prime}\right)$ on $Y$, with $\operatorname{det}\left(\frac{\partial R_{i}}{\partial y_{j}}\right) \neq 0$, such that $\pi_{*} X_{G_{i}^{e}}=\frac{\partial}{\partial y_{i}^{\prime}}, i=1, \ldots, k$.

Since $\left\{G_{i}^{e}, G_{j}^{e}\right\}=0$ and because of $(5.12)$ we can even take (local) canonical coordinates $\left(y^{\prime}, v\right)=\left(y_{1}^{\prime}, \ldots, y_{m}^{\prime}, v_{1}, \ldots, v_{m}\right)$ for $T^{*} Y$ such that

$$
G_{i}^{e}=v_{i} \quad i=1, \ldots, k
$$

(This follows from Darboux's theorem. See for similar arguments van der Schaft (1981, Th. 3.3), (1983c, Th. 2.7)). The submanifold $v_{1}=\cdots=v_{m}=0$ is a Lagrangian submanifold of $T^{*} Y$, and therefore has a generating function $P\left(y_{1}, \ldots, y_{m}\right)$, i.e.

$$
\begin{aligned}
& \left\{\left(y_{1}^{\prime}, \ldots, y_{m}^{\prime}, v_{1}, \ldots, v_{m}\right) \mid v_{1}=\ldots=v_{m}=0\right\} \\
& \quad=\left\{\left(y_{1}, \ldots, y_{m}, u_{1}, \ldots, u_{m}\right) \mid u_{j}=\frac{\partial P}{\partial y_{j}}(y), j=1, \ldots, m\right\}
\end{aligned}
$$

In the new canonical coordinates $\left(y^{\prime}, v\right)$ for $T^{*} Y$ the $F_{i}^{e}$ are of the form

$$
F_{i}^{e}\left(y^{\prime}, v, z\right)=\sum_{j=1}^{k} v_{j} K_{j}^{i i}(z)+V^{\prime i}(z)
$$

since $F_{i}^{e}$ is affine in $v$ and by condition 2

$$
\begin{aligned}
\operatorname{span}_{i=1, \ldots, n}\left\{\sum_{j=1}^{m}\left(\frac{\partial F_{i}^{e}}{\partial y_{j}^{\prime}} d y_{j}^{\prime}+\frac{\partial F_{i}^{e}}{\partial v_{j}} d v_{j}\right)\right\} & =\operatorname{span}_{i=1, \ldots, n}\left\{d G_{i}^{e}\right\} \\
& =\operatorname{span}\left\{d v_{1}, \ldots, d v_{k}\right\}
\end{aligned}
$$

in every point $\left(y^{\prime}, v, z\right)$. However since $F_{i}^{e}\left(y^{\prime}, v, z\right)$ is determined by

$$
\left\{H-P \circ C-\sum_{j=1}^{m} v_{j} C_{j}^{\prime}, F_{i}\right\}(x)=F_{i}^{e}\left(C^{\prime}(x), v, F(x)\right)
$$

where $C^{\prime}=\left(C_{1}^{\prime}, \ldots, C_{m}^{\prime}\right)$ in the new coordinates $\left(y_{1}^{\prime}, \ldots, y_{m}^{\prime}\right)$ for $Y$, it follows from (5.16) that

$$
\begin{array}{ll}
\left\{H-P \circ C, F_{i}\right\}(x)=V^{\prime i}(F(x)) & i=1, \ldots, n \\
\left\{C_{j}^{\prime}, F_{i}\right\}(x)=-K_{j}^{\prime i}(F(x)) & i=1, \ldots, n \\
& j=1, \ldots, m
\end{array}
$$


or equivalently

$$
\begin{aligned}
& \{H-P \circ C, \mathscr{F}\} \subset \mathscr{F} \\
& \left\{R_{j} \circ C, \mathscr{F}\right\} \subset \mathscr{F} \quad j=1, \ldots, m
\end{aligned}
$$

as was to be proved.

Conversely, suppose that $(5.19)=(5.1)$ holds. Then

$$
\left\{C_{j}, F_{i}\right\}=\left\{\left(R^{-1}\right)_{j} \circ R \circ C, F_{i}\right\}=\sum_{k=1}^{m} \frac{\partial\left(R^{-1}\right) j}{\partial y_{k}^{\prime}}(R \circ C)\left\{F_{k} \circ C, F_{i}\right\}
$$

with $R: Y \rightarrow Y$ defined by $R=\left(R_{1}, \ldots, R_{m}\right)$. Since $\left\{R_{k} \circ C, F_{i}\right\}=-K_{k}^{i} \in \mathscr{F}$ it follows that $\left\{C_{j}, F_{i}\right\} \in \mathscr{F}+\mathscr{C}, \forall i, j$. Also, since $\left\{H-P \circ C, F_{i}\right\}=V^{i} \in \mathscr{F}$ we have

$$
\left\{H, F_{i}\right\}=\left\{H-P \circ C, F_{i}\right\}+\left\{P \circ C, F_{i}\right\}=V^{\prime i}+\sum_{j=1}^{m} \frac{\partial\left(P \circ R^{-1}\right)}{\partial y_{j}^{\prime}}(R \circ C),
$$

and

$$
\left\{R_{j} \circ C, F_{i}\right\}=V^{\prime i}+\sum_{j=1}^{m} \frac{\partial\left(P \circ R^{-1}\right)}{\partial y_{j}^{\prime}}(R \circ C) K_{j}^{i} \in \mathscr{F}+\mathscr{C}
$$

Hence for $i=1, \ldots, n$

$$
\begin{aligned}
F_{i}^{e}(y, u, z)= & V^{\prime i}(z)+\sum_{j=1}^{m} \frac{\partial\left(P \circ R^{-1}\right)}{\partial y_{j}^{\prime}}(R(y)) K_{j}^{\prime i}(z) \\
& +\sum_{j=1}^{m} u_{j}\left(\sum_{k=1}^{m} \frac{\partial\left(R^{-1}\right) j}{\partial y_{k}^{\prime}}(R(y)) K_{j}^{\prime i}(z)\right)
\end{aligned}
$$

and we may define $G_{i}^{e}(y, u), i=1, \ldots, n$ as

$$
G_{i}^{e}(y, u, z)=\sum_{j=1}^{m} \frac{\partial\left(P \circ R^{-1}\right)}{\partial y_{j}^{\prime}}(R(y))+\sum_{j=1}^{m} u_{j}\left(\sum_{k=1}^{m} \frac{\partial\left(R^{-1}\right)_{j}}{\partial y_{k}^{\prime}}(R(y))\right)
$$

Then it is clear that condition 2 is satisfied.

Remark 1. Condition 2 is a direct specialization of the integrability condition for output feedback derived in Nijmeijer \& van der Schaft (1982a, Theorem 3.1, 3.2 , condition iii) to Hamiltonian systems. In this reference one seeks for conditions to make a distribution $D$ for a general nonlinear system $\dot{x}=$ $f(x, u), y=h(x)$ invariant by means of output feedback. One defines the codistribution $P$ on $M$ by $\operatorname{Ker} P=D$. Then the prolonged codistribution $\dot{P}$ on $T M$ has 
to be such that the codistribution $\operatorname{span}\left\{P, f^{*} \dot{P}\right\} \cap \operatorname{span}\{d h, d u\}$ is involutive. In the Hamiltonian case $D=D_{\mathscr{F F}}$, and if $\mathscr{F}$ is Lagrangian $P=d \mathscr{F}$. Furthermore since $f(x, u)=X_{H}(x)-\sum_{j=1}^{m} u_{j} X_{C_{j}}(x)$ we have for $\dot{F}_{i} \in \dot{\mathscr{F}}$

$$
\begin{aligned}
f^{*} \dot{F}_{i} & =\dot{F}_{i} \circ\left(X_{H}(x)-\sum_{j=1}^{m} u_{j} X_{C_{j}}(x)\right)=\left(X_{H}-\sum_{j=1}^{m} u_{j} X_{C_{j}}\right)\left(F_{i}\right)(x) \\
& =\left\{H-\sum_{j=1}^{m} u_{j} C_{j}, F_{i}\right\}(x)=F_{i}^{e}(C(x), u, F(x))
\end{aligned}
$$

Therefore

$$
\begin{aligned}
\operatorname{span}\left\{P, f^{*} \dot{P}\right\} \cap \operatorname{span}\{d h, d u\}= & \operatorname{span}\left\{d \mathscr{F}, d\left\{H-\sum_{j=1}^{m} u_{j} C_{j}, F_{i}\right\}\right\} \\
& \cap \operatorname{span}\{d h, d u\} \\
= & \operatorname{span}\left\{d z, d \mathscr{F}^{e}\right\} \cap \operatorname{span}\{d y, d u\}
\end{aligned}
$$

Now the distribution $\operatorname{span}\left\{d \mathscr{F}^{e}, d z\right\} \cap \operatorname{span}\{d y, d u\}$ on $T^{*} Y \times \mathbb{R}^{n}$ is involutive if and only if condition 2 is satisfied. This follows from the following Lemma which we state without proof.

Lemma 5.3. Let $x=\left(x_{1}, \ldots, x_{n}\right)$ and let $f_{1}(x), \ldots, f_{k}(x)$ be functions. Then for $k$, $r \leqslant n: \operatorname{span}\left\{d f_{1}(x), \ldots, d f_{k}(x), d x_{r+1}, \ldots, d x_{m}\right\} \cap \operatorname{span}\left\{d x_{1}, \ldots, d x_{r}\right\}$ is involutive, if and only if there exist functions $g_{1}\left(x_{1}, \ldots, x_{r}\right), \ldots, g_{k}\left(x_{1}, \ldots, x_{r}\right)$ such that

$$
\underset{i=1, \ldots, k}{\operatorname{span}}\left\{\sum_{l=1}^{r} \frac{\partial f_{i}}{\partial x_{l}} d x_{l}\right\}=\operatorname{span}_{i=1, \ldots, k}\left\{\sum_{l=1}^{r} \frac{\partial g_{i}}{\partial x_{l}} d x_{l}\right\} .
$$

Remark 2. Condition 2 can be equivalently formulated in the following way. Denote the projection from $T^{*} Y \times \mathbb{R}^{n}$ to $T^{*} Y$ by $p$. Then condition 2 is equivalent to: $D_{\mathscr{F} \mathcal{F}^{e}}=\operatorname{span}\left\{X_{F_{1}^{e}}, \ldots, X_{F_{n}^{e}}\right\}$ (distribution on $\left.T^{*} Y \times \mathbb{R}^{n} !\right)$ projects under $p$ to a distribution on $T^{*} Y^{n}$. In fact $p_{*} D_{\mathscr{g F}}$ should equal $D_{\mathscr{G} e}=$ $\operatorname{span}\left\{X_{G^{e}}, \ldots, X_{G_{n}^{e}}\right\}$ (a distribution on $T^{*} Y$ ). Now we know from van der Schaft (1982a) that $D_{\mathscr{F}{ }^{\circ}}$ projects to a distribution on $T^{*} Y$ if and only if

$$
\left[\frac{\partial}{\partial z_{i}}, X_{F_{j}}\right] \subset \operatorname{span}\left\{\frac{\partial}{\partial z_{i}}, \ldots, \frac{\partial}{\partial z_{m}}\right\}+D_{\mathscr{F} \cdot} \quad \begin{aligned}
& i=1, \ldots, n \\
& j=1, \ldots, n
\end{aligned}
$$

Let us give a simple (mathematical) example of the above theorem:

Example 5.4. Consider the Hamiltonian system on

$$
M=\left\{\left(q_{1}, q_{2}, p_{1}, p_{2}\right) \mid p_{1}+p_{2} \neq 0\right\}
$$


with

$$
\begin{aligned}
H= & \frac{1}{\left(p_{1}+p_{2}\right)}+\frac{1}{4}\left(q_{1}-q_{2}\right)^{2}+\left(p_{1}+p_{2}\right) \sin q_{1} \cos q_{2} \\
& +\left(p_{1}+p_{2}\right) \cos q_{1} \sin q_{2} \\
C_{1}= & \left(p_{1}+p_{2}\right) \cos \frac{1}{2}\left(q_{1}-q_{2}\right) \\
C_{2}= & \left(p_{1}+p_{2}\right) \sin \frac{1}{2}\left(q_{1}-q_{2}\right)
\end{aligned}
$$

and output manifold $Y=\mathbb{R}^{2} \backslash\{0\}$. Then $\mathscr{F}:=\operatorname{span}\left\{q_{1}+q_{2}, p_{1}-p_{2}\right\}$ is a Lagrangian function group. Choose new canonical coordinates $P_{1}=q_{1}+q_{2}, P_{2}=$ $p_{1}-p_{2}, Q_{1}=-\frac{1}{2}\left(p_{1}+p_{2}\right), Q_{2}=\frac{1}{2}\left(q_{1}-q_{2}\right)$. It easily follows that $\mathscr{F}$ is l.c.i. Furthermore $\mathscr{F}$ is also l.h.c.i. as can be seen as follows. First apply Hamiltonian feedback $V_{1}\left(y_{1}, y_{2}\right)=\left(y_{1}^{2}+y_{2}^{2}\right)^{-1 / 2}$. Then $V_{1} \circ C=\frac{1}{p_{1}+p_{2}}$. Furthermore, since $C_{1}=-2 Q_{1} \cos Q_{2}, C_{2}=-2 Q_{1} \sin Q_{2}$, there exist $R_{1}$ and $R_{2}$ on $Y$ such that $R_{1} \circ C=Q, R_{2} \circ C=Q_{2}$. Then it is clear that $\left\{R_{1} \circ C, \mathscr{F}\right\} \subset \mathscr{F},\left\{R_{2} \circ C, \mathscr{F}\right\} \subset$ $\mathscr{F}$. Finally apply feedback $V_{2}\left(y_{1}^{\prime}, y_{2}^{\prime}\right)=\left(y_{2}^{\prime}\right)^{2}$ then $\left\{H-V_{1}\left(C_{1}, C_{2}\right)-\right.$ $\left.V_{2}\left(R_{1} \circ C, R_{2} \circ C\right), \mathscr{F}\right\} \subset \mathscr{F}$, so $\mathscr{F}$ is made invariant by Hamiltonian feedback.

If there exists a Lagrangian function group $\mathscr{F}$ satisfying (5.1) we obtain the following normal form for the feedback transformed Hamiltonian system. By Theorem 1.1. there exists local canonical coordinates $\left(q_{1}, \ldots, q_{n}, p_{1}, \ldots, p_{n}\right)$ such that $\mathscr{F}=\operatorname{span}\left\{p_{1}, \ldots, p_{n}\right\}$. Then (5.1) implies

$$
\frac{\partial(H-P \circ C)}{\partial q_{i}} \in \mathscr{F}, \frac{\partial\left(R_{j} \circ C\right)}{\partial q_{i}} \in \mathscr{F} \quad \begin{aligned}
& i=1, \ldots, n \\
& j=1, \ldots, m
\end{aligned}
$$

Hence $H-P \circ C$ and $R_{j} \circ C$ are of the form

$$
\begin{aligned}
& H-P \circ C=\sum_{i=1}^{n} h_{i}(p) q_{i}+h(p) \\
& R_{j} \circ C=\sum_{i=1}^{n} c_{i}^{j}(p) q_{i}+c^{j}(p) \quad j=1, \ldots, m
\end{aligned}
$$

and the feedback transformed system equals

$$
\begin{aligned}
& \dot{q}_{i}=\sum_{k=1}^{n} \frac{\partial h_{k}(p)}{\partial p_{i}} q_{k}+\frac{\partial h(p)}{\partial p_{i}}-\sum_{j=1}^{m} v_{j} \sum_{k=1}^{n} \frac{\partial c_{k}^{j}(p)}{\partial p_{i}} q_{k}+\frac{\partial c^{j}(p)}{\partial p_{i}} \\
& \dot{p}_{i}=-h_{i}(p)+\sum_{j=1}^{m} v_{j} c_{i}^{j}(p) \quad i=1, \ldots, n
\end{aligned}
$$

Notice furthermore the close relationship with the notion of complete integrability of a Hamiltonian vectorfield $X_{H} . X_{H}$ is completely integrable if there exist $n$ independent functions $F_{1}, \ldots, F_{n}$ such that $\left\{H, F_{i}\right\}=0$ and $\left\{F_{i}, F_{j}\right\}=0, i, j=$ 
$1, \ldots, n$. It follows that $\mathscr{F}:=\operatorname{span}\left\{F_{1}, \ldots, F_{n}\right\}$ is a Lagrangian function group satisfying $\{H, \mathscr{F}\}=0$.

We remark that the construction of action-angle coordinates used in the context of such completely integrable Hamiltonian vectorfields can be immediately applied to Lagrangian invariant function groups. This yields a sort of global interpretation of the local normal form (5.25) (see Abraham \& Marsden (1978)).

Remark. Although we have confined ourselves to affine Hamiltonian systems (1.4), the developed theory of local controlled invariance can be extended to general Hamiltonian systems given by a generating function $H(q, p, u)$, as treated in Brockett (1977), van der Schaft (1982a, b, 1983b). In this case a function group $\mathscr{F}=\operatorname{span}\left\{F_{1}, \ldots, F_{k}\right\}$ is called 1.c.i. if there exist functions $F_{i}^{e}$ on $T^{*} Y \times \mathbb{R}^{k}$ such that

$$
\left\{H(q, p, u), F_{i}\right\}=F_{i}^{e}\left(-\frac{\partial H}{\partial u}(q, p, u), u, F(q, p)\right) \quad i=1, \ldots, k
$$

where $F:=\left(F_{1}, \ldots, F_{k}\right)$. So the only difference with (5.7) is that the $F_{i}^{e}$ need no longer to be affine in $u$. Furthermore if $\mathscr{F}$ is Lagrangian it can be proved (cf. van der Schaft (1984b), Theorem 5) that the mapping $F_{i} \rightarrow F_{i}^{e}$ is a Poisson algebra morphism, and so $\left\{F_{i}, F_{j}\right\}_{T^{*} Y \times \mathbf{R}^{k}}=0, \forall i, j$, as in Theorem 5.2.

\section{Invariance by Dynamic Hamiltonian Feedback}

In the previous section we have seen that under an integrability condition and some regularity assumptions a Lagrangian function group $\mathscr{F}$ locally can be made invariant by Hamiltonian feedback if $\mathscr{F}$ is locally controlled invariant. In this section we will show how we can extend this procedure to coisotropic function groups. The trick is to augment the state space of the system and to lift the coisotropic function group to a Lagrangian function group on the augmented state space. Hamiltonian feedback for this augmented system corresponds to dynamic Hamiltonian feedback for the original system.

Definition 6.1. Let $\mathbb{R}^{2 l}$ be endowed with its natural symplectic form. We define the auxiliary Hamiltonian system on $\mathbb{R}^{2 l}$ as

$$
\begin{array}{rlrl}
\dot{\xi}_{i}=-\nu_{i} & & i=1, \ldots, l \\
\dot{\xi}_{i}=\nu_{i} & i & =l+1, \ldots, 2 l \\
\eta_{i}=\xi_{i} & i & =1, \ldots, 2 l
\end{array}
$$

where $\xi=\left(\xi_{1}, \ldots, \xi_{2 l}\right)$ are the standard coordinates for the state space $M^{a u}:=$ $\mathbb{R}^{2 l}, \nu=\left(\nu_{1}, \ldots, \nu_{2 l}\right)$ are the standard coordinates for the input space $U^{a u}:=\mathbb{R}^{2 l}$ and $\eta=\left(\eta_{1}, \ldots, \eta_{2 l}\right)$ are the standard coordinates for the output space $Y^{a u}:=\mathbb{R}^{2 l}$.

Given a Hamiltonian system (1.4) with state space $(M, \omega)$ and output space $Y$ the augmented Hamiltonian system is defined as the product Hamiltonian system 
of (1.4) and (6.1):

$$
\begin{array}{ll}
\dot{x}=X_{H}(x)-\sum_{j=1}^{m} u_{j} X_{C_{j}}(x) & \\
\dot{\xi}_{i}=-\nu_{i} & i=1, \ldots, l \\
\dot{\xi}_{i}=\nu_{i} & i=l+1, \ldots, 2 l \\
y_{j}=C_{j}(x) & j=1, \ldots, m \\
\eta_{i}=\xi_{i} & i=1, \ldots, 2 l
\end{array}
$$

with state space $M^{a}=M \times M^{a \mu}$, output space $Y^{a}=Y \times Y^{a u}$, and input-output space $T^{*} Y^{a}=T^{*} Y \times Y^{a u} \times U^{a u}$. Denoting $x^{a}=(x, \xi), y^{a}=(y, \eta), u^{a}=(u, \nu)$, the augmented Hamiltonian system has an internal energy $H^{a}(x, \xi)=H(x)$ and output maps

$$
\begin{array}{ll}
C_{j}^{a}(x, \xi)=C_{j}(x) & j=1, \ldots, m \\
C_{j}^{a}(x, \xi)=\xi_{i} & j=m+1, \ldots, m+2 l
\end{array}
$$

Dynamic Hamiltonian feedback for (1.4) is static Hamiltonian feedback for the augmented system (6.2), i.e. there exist functions $P^{a}(y, \eta), R_{1}^{a}(y, \eta)$, $\ldots, R_{m+2 l}^{a}(y, \eta)$, with $\left(R_{1}^{a}, \ldots, R_{m+2 l}^{a}\right): Y \times \mathbb{R}^{2 l} \rightarrow Y \times \mathbb{R}^{2 l}$ a diffeomorphism, such that the augmented system is transformed into

$$
\dot{x}^{a}=X_{H^{a}-P^{a} \circ C^{a}}\left(x^{a}\right)-\sum_{j=1}^{m} u_{j} X_{R_{j}^{a} \circ C^{a}}\left(x^{a}\right)-\sum_{j=m+1}^{m+2 l} \nu_{j} X_{R_{j}^{a} \circ C^{a}}\left(x^{a}\right)
$$

Remark. Of course, the auxiliary system (6.1) corresponds (modulo minus signs) to $2 l$ independent integrators.

The main theorem of this section reads as follows

Theorem 6.2. Let (1.4) be a Hamiltonian system on $(M, \omega)$, and let $\mathscr{F}$ be $a$ coisotropic function group on $M$, satisfying Condition A. Thus $F=$ $\operatorname{span}\left\{F_{1}, \ldots, F_{k}\right\}, F_{i}$ independent, $k \geqslant n$. Suppose satisfies

1) $\mathscr{F}$ is locally controlled invariant, so there exist functions $F_{1}^{e}, \ldots, F_{k}^{e}: T^{*} Y \times$ $\mathbb{R}^{k} \rightarrow \mathbb{R}$ such that

$$
\left\{H-\sum_{j=1}^{m} u_{j} C_{j}, F_{i}\right\}(x)=F_{i}^{e}(C(x), u, F(x)), \quad i=1, \ldots, k
$$

(again we denote $F=\left(F_{1}, \ldots, F_{k}\right): M \rightarrow \mathbb{R}^{k}$ )

2) The function space. $\mathscr{F}^{e}:=\operatorname{span}\left\{F_{1}^{e}, \ldots, F_{k}^{e}\right\}$ on $T^{*} Y \times \mathbb{R}^{k}$ "projects" to a function space on $T^{*} Y$, i.e. there are functions $G_{1}^{e}, \ldots, G_{k}^{e}: T^{*} Y \rightarrow \mathbb{R}$ such that

$$
\underset{i=1, \ldots, k}{\operatorname{span}}\left\{\sum_{j=1}^{m} \frac{\partial F_{i}^{e}}{\partial y_{j}} d y_{j}+\frac{\partial F_{i}^{e}}{\partial u_{j}} d u_{j}\right\}=\operatorname{span}_{i=1, \ldots, k}\left\{d G_{i}^{e}\right\}
$$


in every point $(y, u, z)$ of $T^{*} Y \times \mathbb{R}^{k}$

3) The function spaces $\mathscr{C}, \mathscr{F}+\mathscr{C}$ satisfy Condition A

4) The function spaces $\mathscr{F}^{e}$ and $\mathscr{G}^{e}:=\operatorname{span}\left\{G_{1}^{e}, \ldots, G_{k}^{e}\right\}$ satisfy Condition A.

5) The codistribution $\operatorname{span}_{i=1, \ldots, k}\left\{\frac{\partial F_{i}^{e}}{\partial u_{j}} d u_{j}\right\}$ on $T^{*} Y \times \mathbb{R}^{k}$ has constant dimension.

Then, there exists locally a Lagrangian function group $\mathscr{F}_{\text {lift }}$ on the augmented space $M \times \mathbb{R}^{2 l}$, with $l=k-n$, such that if the projection $M \times \mathbb{R}^{2 l} \rightarrow M$ is denoted by $\pi$, then

$$
\begin{aligned}
& \pi_{*} D_{\mathscr{F}_{\text {lift }}}=D_{\mathscr{F}}, D_{\mathscr{F}_{\text {lift }}} \cap(T M \times 0)=D_{\mathscr{F} \perp} \\
& \operatorname{dim} d \mathscr{F}_{\text {lift }}=\operatorname{dim} d \mathscr{F}
\end{aligned}
$$

( $T M \times 0$ is the distribution on $M \times \mathbb{R}^{2 l}$ with zero-components in the $\mathbb{R}^{2 l}$-direction). $\mathscr{F}_{\text {lift }}$ is called the lift of $\mathscr{F}$. Moreover there exists locally a dynamic Hamiltonian feedback $\left(P^{a}(y, \xi), R_{1}^{a}(y, \xi), \ldots, R_{m+2 l}^{a}(y, \xi)\right)$, with $\xi \in \mathbb{R}^{2 l}$ such that

$$
\begin{aligned}
& \left\{H^{a}-P^{a} \circ C^{a}, \mathscr{F}_{\text {lift }}\right\} \subset \mathscr{F}_{\text {lift }} \\
& \left\{R_{j}^{a} \circ C, \mathscr{F}_{\text {lift }}\right\} \subset \mathscr{F}_{\text {lift }} \quad j=1, \ldots, m+2 l
\end{aligned}
$$

Conversely, if (6.6) holds, then conditions 1 and 2 are satisfied.

In order to construct the function group $\mathscr{F}_{\text {lift }}$ we need the following lemma, which follows from Theorem 1.1. but also can be proved directly.

Lemma 6.3. Let $\mathscr{F}$ be a coisotropic function group on $(M, \omega)$, with $\operatorname{dim} d \mathscr{F}(x)=$ $k \geqslant n=\frac{1}{2} \operatorname{dim} M, \forall x$. Then there exist local canonical coordinates $\left(q_{1}, \ldots, q_{n}, p_{1}, \ldots, p_{n}\right)$ for $M$ such that locally $\mathscr{F}=\operatorname{span}\left\{q_{1}, \ldots, q_{k-n}, p_{1}, \ldots, p_{n}\right\}$.

Proof. Consider the function group $\mathscr{F} \perp$. Take $2 n-k$ independent functions $p_{k-n+1}, \ldots, p_{n}$ such that $\mathscr{F}^{\perp}=\operatorname{span}\left\{p_{k-n+1}, \ldots, p_{n}\right\}$. Since $\mathscr{F} \perp \subset \mathscr{F}$ we have $\left\{p_{i}, p_{j}\right\}=0$ for every $i, j=k-n+1, \ldots, n$.

Hence $p_{k-n+1}, \ldots, p_{n}$ are a set of partial canonical coordinates. By Darboux's theorem (see the proof in Weinstein (1983)) we can extend the set $\left(p_{k-n+1}, \ldots, p_{n}\right)$ to a set $(q, p)=\left(q_{1}, \ldots, q_{n}, p_{1}, \ldots, p_{n}\right)$ of canonical coordinates. Now consider a function $F(q, p)$ contained in $\mathscr{F}$. Since $\mathscr{F}^{\perp}=\operatorname{span}\left\{p_{k-n+1}, \ldots, p_{n}\right\}$ we have $0=-\left\{F, p_{i}\right\}=\frac{\partial F}{\partial q_{i}}, i=k-n+1, \ldots, n$. Hence $F$ is only a function of $\left(q_{1}, \ldots, q_{k-n}, p_{1}, \ldots, p_{n}\right)$. Since $\operatorname{dim} d \mathscr{F}=k$ it follows that $\mathscr{F}=$ $\operatorname{span}\left\{q_{1}, \ldots, q_{k-n}, p_{1}, \ldots, p_{n}\right\}$.

Proof of Theorem 6.2. Let $\mathscr{F}$ be a coisotropic function group for which conditions 1 up till 5 are satisfied. Choose local canonical coordinates for $\mathscr{F}$ as in Lemma 6.3., i.e. $\mathscr{F}=\operatorname{span}\left\{q_{1}, \ldots, q_{l}, p_{1}, \ldots, p_{n}\right\}$, with $l=k-n$. The state space of the auxiliary Hamiltonian system will be $\mathbb{R}^{2 l}$ with its usual symplectic form. Define locally the lifted function group $\mathscr{F}_{\text {lift }}$ on $M \times \mathbb{R}^{2 l}$ as

$$
\mathscr{F}_{\text {lift }}=\operatorname{span}\left\{q_{1}+\xi_{l+1}, \ldots, q_{l}+\xi_{2 l}, p_{1}+\xi_{1}, \ldots, p_{l}+\xi_{l}, p_{l+1}, \ldots, p_{n}\right\}
$$


with $\xi=\left(\xi_{1}, \ldots, \xi_{2 l}\right) \in \mathbb{R}^{2 l}$. Since

$$
\left\{q_{i}+\xi_{l+i}, p_{i}+\xi_{i}\right\}_{M \times \mathbf{R}^{2 l}}=\left\{q_{i}, p_{i}\right\}_{M}+\left\{\xi_{l+i}, \xi_{i}\right\}_{\mathbf{R}^{2 l}}=-1+1=0,
$$

$i=1, \ldots, l$, it follows that for any $F_{1}, F_{2} \in \mathscr{F}_{\text {lift }}$

$$
\left\{F_{1}, F_{2}\right\}_{M \times \mathbf{R}^{2 l}}=0
$$

Since $\operatorname{dim} d \mathscr{F}_{\text {lift }}=n+l=\frac{1}{2} \operatorname{dim}\left(M \times \mathbb{R}^{2 l}\right)$ this implies that $\mathscr{F}_{\text {lift }}$ is a Lagrangian function group on $M \times \mathbb{R}^{2 l}$ (Of course $\mathscr{F}_{\text {lift }}$ is only defined in a neighborhood of every point in $M$ ). Since $\mathscr{F}$ is locally controlled invariant it follows that

$$
\begin{aligned}
& \left\{H^{a}, \mathscr{F}_{\text {lift }}\right\} \subset \mathscr{F}_{\text {lift }}+\mathscr{C}^{a} \\
& \left\{\mathscr{C}^{a}, \mathscr{F}_{\text {lift }}\right\} \subset \mathscr{F}_{\text {lift }}+\mathscr{C}^{a}
\end{aligned}
$$

Hence, $\mathscr{F}_{\text {lift }}$ is locally controlled invariant for the augmented Hamiltonian system. Furthermore we have

$$
\begin{aligned}
& \left\{H^{a}-\sum_{j=1}^{m} u_{j} C_{j}^{a}-\sum_{k=1}^{2 l} \nu_{k} C_{k}^{a}, q_{i}+\xi_{l+i}\right\} \\
& =\left\{H-\sum_{j=1}^{m} u_{j} C_{j}-\sum_{k=1}^{2 l} \nu_{k} \xi_{k}, q_{i}+\xi_{l+i}\right\} \\
& =\left\{H-\sum_{j=1}^{m} u_{j} C_{j}, q_{i}\right\}-\sum_{k=1}^{2 l} \nu_{k}\left\{\xi_{k}, \xi_{l+i}\right\} \\
& =\left\{H-\sum_{j=1}^{m} u_{j} C_{j}, q_{i}\right\}+\nu_{i} \quad i=1, \ldots, l
\end{aligned}
$$

and

$$
\begin{aligned}
&\left\{H^{a}-\sum_{j=1}^{m} u_{j} C_{j}^{a}-\sum_{k=1}^{2 l} \nu_{k} C_{k}^{a}, p_{i}+\xi_{i}\right\}=\left\{H-\sum_{j=1}^{m} u_{j} C_{j}, p_{i}\right\}-\sum_{k=1}^{2 l} \nu_{k}\left\{\xi_{k}, \xi_{i}\right\} \\
&=\left\{H-\sum_{j=1}^{m} u_{j} C_{j}, p_{i}\right\}-\nu_{l+i} \\
& i=1, \ldots, l
\end{aligned}
$$

and

$$
\begin{aligned}
&\left\{H^{a}-\sum_{j=1}^{m} u_{j} C_{j}^{a}-\sum_{k=1}^{2 l} \nu_{k} C_{k}^{a}, p_{l+i}\right\}=\left\{H-\sum_{j=1}^{m} u_{j} C_{j}, p_{l+i}\right\} \\
& i=1, \ldots, n-l
\end{aligned}
$$


The functions $\left\{H-\sum_{j=1}^{m} u_{j} C_{j}, q_{i}\right\}, i=1, \ldots, l$ and $\left\{H-\sum_{j=1}^{m} u_{j} C_{j}, p_{i}\right\}, i=1, \ldots, n$ are all contained in $\mathscr{F}^{e}$. It is now easily seen that conditions $2,3,4,5$ imply conditions 2,3,4,5 of Theorem 5.2. for the augmented Hamiltonian system. Hence by Theorem 5.2. there exist (locally on $Y \times \mathbb{R}^{2 l}$ ) functions $P^{a}(y, \eta), R_{1}^{a}(y, \eta), \ldots, R_{m+2 l}^{a}(y, \eta)$, where the Jacobian of $\left(R_{1}^{a}, \ldots, R_{m+2 l}^{a}\right)$ has full rank, such that (6.6) is satisfied. The proof of the converse statement follows the same lines as the proof of the converse statement in Theorem 5.2.

Example 6.4. Consider the Hamiltonian system from Example 2.5 with $H(q, p)$ $=\frac{1}{2} e^{q_{2}} p_{1}^{2}+\frac{1}{2} p_{2}^{2}+\frac{1}{2} q_{1}^{2}+\frac{1}{2} q_{2}^{2}, C(q, p)=q_{1}$. Consider the velocity $\dot{q}_{1}=\frac{\partial H}{\partial p_{1}}=$ $e^{q_{2}} p_{1}$. Define $\mathscr{F}:=\left(\operatorname{span}\left\{e^{q_{2}} p_{1}\right\}\right)^{\perp}=\operatorname{span}\left\{q_{2}, p_{2}+q_{1} p_{1}, p_{1}\right\}$. Since $\operatorname{span}\left\{e^{q_{2}} p_{1}\right\} \subset \mathscr{F}, \mathscr{F}$ is coisotropic. Furthermore since $\mathscr{F}+\operatorname{span}\left\{q_{1}\right\}=$ $\operatorname{span}\left\{q_{1}, q_{2}, p_{1}, p_{2}\right\}, \mathscr{F}$ is 1.c.i. Let us therefore try to construct a dynamic Hamiltonian feedback which makes $\mathscr{F}$ invariant. First we have to choose canonical coordinates $\left(Q_{1}, Q_{2}, P_{1}, P_{2}\right)$ such that $\mathscr{F}=\operatorname{span}\left\{Q_{1}, P_{1}, P_{2}\right\}$. Since the Poisson structure (1.17) associated with $\mathscr{F}$ is

$$
\left(w_{i j}\right)=\left(\begin{array}{rrr}
0 & -1 & 0 \\
1 & 0 & -p_{1} \\
0 & p_{1} & 0
\end{array}\right)
$$

we take $Q_{1}=q_{2}, P_{1}=p_{2}+q_{1} p_{1}, P_{2}=\log p_{1}+q_{2}, Q_{2}=q_{1} p_{1}$ (assume $p_{1}>0$ ). The augmented state space will be $Q_{1}, Q_{2}, P_{1}, P_{2}, \xi_{1}, \xi_{2}$ and $\mathscr{F}_{\text {lift }}=\operatorname{span}\left\{Q_{1}+\right.$ $\left.\xi_{2}, P_{1}+\xi_{1}, P_{2}\right\}$. However although $\mathscr{F}_{\text {lift }}$ is 1.c.i. and Lagrangian, it cannot be made invariant by Hamiltonian feedback. This is because the integrability condition 2 is not satisfied (because of the $q_{1} p_{1}$-term in $\mathscr{F}$, while $C=q_{1}$ )

\section{Application to Disturbance Decoupling}

Probably the easiest application of controlled invariance is the problem of disturbance decoupling. Consider a Hamiltonian system (1.4) with additional disturbances

$$
\begin{aligned}
& \dot{x}=X_{H}(x)-\sum_{j=1}^{m} u_{j} X_{C_{j}}(x)-\sum_{k=1}^{l} d_{k} E_{k}(x) \\
& y_{j}=C_{j}(x) \quad j=1, \ldots, m
\end{aligned}
$$

The disturbances $d_{1}, \ldots, d_{l}$ enter the equations via the (known) vectorfields $E_{1}, \ldots, E_{l}$. Suppose one has furthermore a set of functions of the state

$$
z_{j}=G_{j}(x) \quad j=1, \ldots, r
$$

where $z_{1}, \ldots, z_{r}$ are the so-called to-be-regulated variables. 
One now wishes to apply Hamiltonian feedback to (7.1) such that after feedback the disturbances do not influence the to-be-regulated variables. This can be done, using Theorems 5.2 and 6.2 , if there exists a locally controlled invariant function group $\mathscr{F}$, contained in the function group $\mathscr{G}^{\perp}=\left(\operatorname{span}\left\{G_{1}, \ldots, G_{r}\right\}\right)^{\perp}$, which is Lagrangian or coisotropic, and such that the distribution $\operatorname{span}\left\{E_{1}, \ldots, E_{l}\right\}$ is contained in $D_{\mathscr{F} \perp}$ (Recall the construction of $\mathscr{F}_{\text {lift }}$ in (6.5)!). In case $\mathscr{F}$ is Lagrangian, static Hamiltonian feedback is sufficient; otherwise dynamic Hamiltonian feedback is needed. Notice that a necessary condition for the existence of such an $\mathscr{F}$ is that $\mathscr{G}^{\perp}$ itself has to be coisotropic. Indeed let $\mathscr{F} \subset \mathscr{G} \perp$ with $\mathscr{F} \perp \subset \mathscr{F}$, then $\mathscr{G} \subset\left(\mathscr{G}^{\perp}\right)^{\perp} \subset \mathscr{F} \perp \subset \mathscr{F} \subset \mathscr{G}^{\perp}$.

A computational procedure for solving the disturbance decoupling problem by Hamiltonian feedback may be the following:

1. Compute the maximal l.c.i. function group $\mathscr{F}^{*}$ contained in $\mathscr{G}^{\perp}$, using the $\mathscr{F} *$-algorithm (2.4).

2. Check if $\operatorname{span}\left\{E_{1}, \ldots, E_{l}\right\}$ is contained in $D_{\mathscr{F} * 1}$.

3. Check if $\mathscr{F}^{*}$ is Lagrangian or coisotropic.

4. Check if $\mathscr{F}^{*}$ satisfies conditions $2,3,4,5$ of Theorem 5.2 or 6.2 .

Remark. It maybe easier to use the $\mathscr{S}^{*}$-algorithm (4.3) to compute the minimal conditioned invariant function group $\mathscr{S}^{*}$ containing $\mathscr{G}$. Then one may check if $\left(\mathscr{S}^{*}\right)^{\perp}$ is locally controlled invariant (see Proposition 4.4.)

A particularly nice situation arises if the disturbance vectorfields $E_{k}$ are given as the Hamiltonian vectorfields of the functions $G_{j}$, i.e.

$$
E_{k}(x)=X_{G_{k}}(x), k=1, \ldots, r
$$

In this case if a function group $\mathscr{F}$ satisfies $\mathscr{F} \subset \mathscr{G}^{\perp}$, then automatically $\mathscr{G} \subset$ $\left(\mathscr{G}^{\perp}\right)^{\perp} \subset \mathscr{F}^{\perp}$, and so $\operatorname{span}\left\{E_{1}, \ldots, E_{r}\right\}=D_{\mathscr{g}} \subset D_{\mathscr{F}}^{\perp}$. Hence if $\mathscr{F}$ is coisotropic we are done (apart from conditions 2,3,4,5 of Theorem 6.2). In the linear case one can even prove that in this situation disturbance decoupling by general dynamic feedback is possible if and only if there exists a coisotropic $\mathscr{F}$ with $\mathscr{F} \subset \mathscr{G}^{\perp}$ and so if and only if disturbance decoupling by dynamic Hamiltonian feedback is possible.

\section{Conclusion}

The notion of a locally controlled invariant distribution is specialized to Hamiltonian systems by introducing the concept of a locally controlled invariant function group. It is shown that 1.c.i. Lagrangian function groups can be made invariant by Hamiltonian feedback provided an extra integrability condition necessary for the existence of output feedback, is satisfied. This also appears to be the case for symplectic functions groups. Other classes of function groups $\mathscr{F}$ with $\mathscr{F}$ and $\mathscr{F} \perp$ 1.c.i. in general cannot be made invariant by Hamiltonian feedback. However one expects that a "generalized type" of Hamiltonian feedback might work. This is strongly related to the nature of the mapping $F_{i} \rightarrow F_{i}^{e}$ in (5.7), which in this paper is only investigated in the (easy) Lagrangian case. 
Furthermore, it is shown that coisotropic l.c.i. function groups which satisfy the integrability condition can be made invariant by dynamic Hamiltonian feedback.

The developed notion of controlled invariant function groups is only a first, although basic, step in a geometric theory of Hamiltonian control systems. For instance it seems fruitful to combine this notion with the stabilization procedure for Hamiltonian systems as proposed in van der Schaft (1984b).

\section{References}

R. A. Abraham \& J. E. Marsden (1978), Foundations of mechanics, (2nd edition), Benjamin/Cummings, Reading, Mass.

R. W. Brockett (1977), Control theory and analytical mechanics, pp. 1-46 of Geometric control theory (Eds. C. Martin \& R. Hermann), Vol VII of Lie Groups: History, Frontiers and Applications, Math Sci Press, Brookline.

R. Hermann (1977), Toda lattices, cosymplectic manifolds, Bäcklund transformations and kinks. Part A, Interdisciplinary mathematics, Vol. XV, Math Sci Press, Brookline.

R. W. Hirschorn (1981), $(A, B)$-invariant distributions and disturbance decoupling of nonlinear systems, SIAM J. Control \& Opt., 19, 1-19.

A. Isidori, A. J. Krener, C. Gori-Giorgi, S. Monaco (1981a), Nonlinear decoupling via feedback: A differential geometric approach, IEEE Tr. Aut. Control. AC-26, 331-345.

A. Isidori, A. J. Krener, C. Gori-Giorgi, S. Monaco (1981b), Locally $(f, g)$-invariant distributions, Systems \& Control Letters, $1,12-15$.

S. Lie \& F. Engel (1890), Theorie der Transformations Gruppen, Vol II, also Cheisea, New York (1970).

H. Nijmeijer (1981), Controlled invariance for affine control systems, Int. Journal Control, 34, 825-833.

H. Nijmeijer \& A. J. van der Schaft (1982a), Controlled invariance by static output feedback for nonlinear systems, Systems \& Control Letters, 2, 39-47.

H. Nijmeijer \& A. J. van der Schaft (1982b), Controlled invariance for nonlinear systems, IEEE Tr. Aut. Control. AC-27, 904-914.

H. Nijmeijer \& A. J. van der Schaft (1984a), Partial symmetries for nonlinear systems, Memo 459, Twente University of Technology, appeared in Mathematical Systems Theory, 18, 79-96 (1985).

H. Nijmeijer \& A. J. van der Schaft (1984b), Input-output decoupling of Hamiltonian systems: The linear case, Memo 471, Twente University of Technology, appeared in Systems \& Control Letters, 6, 53-58 (1985).

H. Nijmeijer \& A. J. van der Schaft (1984c), Input-output decoupling of Hamiltonian systems: The nonlinear case, in preparation.

A. J. van der Schaft (1981), Symmetries and conservation laws for Hamiltonian systems with inputs and outputs: A generalization of Noether's theorem, Systems \& Control Letters, 1, 295-300.

A. J. van der Schaft (1982a), Observability and controllability for smooth nonlinear systems, SIAM J. Control \& Opt., 20, 338-354.

A. J. van der Schaft (1982b), Hamiltonian dynamics with external forces and observations, Mathematical Systems Theory, 15, 145-168.

A. J. van der Schaft (1983a), Disturbance decoupling by observation feedback for Hamiltonian systems, Systems \& Control Letters, 2, 286-291, 1983.

A. J. van der Schaft (1983b), System theoretic descriptions of physical systems, Doct. Dissertation University of Groningen, 1983, also CWI Tracts No. 3, CWI, Amsterdam, 1984.

A. J. van der Schaft (1983c), Symmetries, conservation laws and time reversibility for Hamiltonian systems with external forces, Journ. of Mathematical Physics, 24, 2095-2101.

A. J. van der Schaft (1984a), Stabilization of Hamiltonian systems, Memo 470, Twente University of Technology, to appear in Journal of Nonlinear Analysis: Theory, Methods and Applications (1985).

A. J. van der Schaft (1984b), Symmetries in optimal control, Memo 491, Twente University of Technology. 
A. J. van der Schaft (1985), On feedback control of Hamiltonian systems, to appear in the Proceedings 7th Int. Symposium on the Mathematical Theory of Networks and Systems (MTNS), Stockholm, June 1985.

A. Weinstein (1983), The local structure of Poisson manifolds, J. Diff. Geometry, 18, 523-557.

W. M. Wonham, (1979), Linear multivariable control: a geometric approach, (2nd edition), Springer, New York.

Received December 18, 1984, and in revised form May 28, 1985, and in final form August 10, 1985. 